\title{
Crosstalk between histone modification and DNA methylation orchestrates the epigenetic regulation of the costimulatory factors, Tim-3 and galectin-9, in cervical cancer
}

\author{
LI ZHANG $^{1}$, SIJUAN TIAN ${ }^{1}$, MEILI PEI ${ }^{1}$, MINYI ZHAO ${ }^{1}$, LI WANG $^{1}$, YIFAN JIANG $^{1}$, \\ TING YANG $^{1}$, JUAN ZHAO ${ }^{1}$, LIHUA SONG ${ }^{2}$ and XIAOFENG YANG ${ }^{1}$
}

\author{
${ }^{1}$ Department of Gynecology and Obstetrics, The First Affiliated Hospital of Xi'an Jiaotong University, Xi'an, Shaanxi 710061; \\ ${ }^{2}$ Research Center for Food Safety and Nutrition, Bor S. Luh Food Safety Research Center, \\ Shanghai Jiao Tong University, Shanghai 200240, P.R. China
}

Received May 31, 2019; Accepted October 10, 2019

DOI: $10.3892 / o r .2019 .7388$

\begin{abstract}
Persistent infection with high-risk human papillomavirus is known to cause cervical cancer. The binding of the costimulatory factors, Tim-3 and galectin-9, can cause immune tolerance and lead to immune escape during carcinogenesis. Epigenetic regulation is essential for Tim-3/galectin-9 expression, which affects the outcome of local cervical cancer infection. Hence, exploring the epigenetic regulatory mechanisms of costimulatory signaling by Tim-3/galectin- 9 is of great interest for investigating the mechanisms through which these proteins are regulated in cervical cancer tumorigenesis. In this study, we report that E2F-1 and FOXM1 mediated by HPV18 E6 and E7 can enhance the transcriptional activity of Enhancer of zeste homolog 2 (EZH2) by binding to its promoter region, resulting in the induced expression of the EZH2-specific target protein, H3K27me3, which consequently reduces the expression of the downstream target gene, DNA (cytosine-5)-methyltransferase 3A (DNMT3A). EZH2 and H3K27me3 directly interact with the DNMT3A promoter region to negatively regulate its expression in HeLa cells. Moreover, the downregulated DNMT3A and the decreased methylation levels in $H A V C R 2 / L G A L S 9$ promoter regions in HeLa cells promoted the expression of Tim-3/galectin-9. Furthermore, the high expression of Tim-3/galectin- 9 was
\end{abstract}

Correspondence to: Professor Xiaofeng Yang, Department of Gynecology and Obstetrics, The First Affiliated Hospital of Xi'an Jiaotong University, 277 West Yanta Road, Xi'an, Shaanxi 710061, P.R. China

E-mail: yxf73@163.com

Dr Lihua Song, Research Center for Food Safety and Nutrition, Bor S. Luh Food Safety Research Center, Shanghai Jiao Tong University, Shanghai 200240, P.R. China

E-mail: lihuas@sjtu.edu.cn

Key words: cervical cancer, HPV18, EZH2, H3K27me3, DNA methylation, Tim-3/galectin-9 associated with HPV positivity among patients with cervical cancer. Moreover, HAVCR2/LGALS9 promoter regions were hypermethylated in normal cervical tissues, and this hypermethylated status inhibited gene expression. On the whole, these findings suggest that EZH2, H3K27me3 and DNMT3A mediate the epigenetic regulation of the negative stimulatory molecules, Tim-3 and galectin-9 in cervical cancer which is associated with HPV18 infection.

\section{Introduction}

Cervical cancer is the most common female malignancy worldwide $(1,2)$. The incidence and mortality rates associated with cervical cancer are particularly higher in developing countries $(3,4)$. It has been estimated that $9.89 / 100,000$ cervical cancer cases and 3.05/100,000 cancer-related deaths occurred in China in 2015 (5). Almost all cervical cancers are caused by persistent infection with high-risk human papillomavirus (HPV), most commonly, HPV16 and HPV18 (6-8).

During carcinogenesis, immune checkpoint pathways are often exploited to evade immune surveillance $(9,10)$, particularly the development of cervical cancer. Hepatitis A virus cellular receptor 2 (HAVCR2) is also known as T-cell immunoglobulin and mucin-domain containing-3 (Tim-3) (11-13). The most important role of Tim-3 is to negatively regulate Th1 immunity. Once Tim-3 binds to its ligand, galectin-9, Tim-3 inhibits Th1 and Th17 responses by hampering their expansion. The Tim-3/galectin-9 pathway contributes to the suppressive tumor microenvironment (TME) in the human body through Treg promotion upon $\mathrm{T}$ cell receptor (TCR) activation $(14,15)$. The DNA methylation of the Tim-3 promoter cooperates with lineage-specific transcription factors in the control of Th cell development $(16,17)$. Cao et al found that the expression of Tim-3 in tumor cells may be an independent prognostic factor for patients with cervical cancer; moreover, Tim-3 expression may promote metastatic potential in cervical cancers (18). Liang et al found that decreased galectin-9 expression was inversely associated with the malignant potential or differentiation of cervical intraepithelial neoplasia (CIN) and cervical squamous cell carcinoma (SCC) as a differentiation 
biomarker (19). Enhancer of zeste homolog 2 (EZH2) mRNA expression has been shown to be increased in cancer tissues compared to normal tissues, and the overexpression of EZH2 has been shown to be associated with the FIGO stage, histological type and lymph node metastasis in cervical cancer (20). The inhibition of DNA (cytosine-5)-methyltransferase 3A (DNMT3A) promotes cervical cancer cell apoptosis, which further demonstrates that DNMT3A is involved in cervical carcinogenesis (21). However, whether the abnormal methylation of the promoter regions of Tim-3/galectin-9 plays a role in the carcinogenesis of cervical cancer remains unclear.

In the present study, it was demonstrated that the promoter regions of $H A V C R 2 / L G A L S 9$ were partially methylated, and that the protein expression of Tim-3/galectin-9 was increased when their methylation level decreased in accordance with the decreased DNMT3A expression. Our previous study found that HPV18 was the second most carcinogenic type of HPV in the Shaanxi Province of China (22). Moreover, HPV18 E6 and E7 participate in the upregulation of EZH2 and H3K27me3 expression, which inhibits DNMT3A expression to downregulate the HAVCR2/LGALS9 methylation levels. We thus hypothesized that the HPV18 oncoproteins E6 and E7 may play an important role in regulating the expression of Tim-3/galectin-9 in the immune microenvironment of cervical cancer through DNA methylation mediated by EZH2/H3K27me3/DNMT3A.

\section{Materials and methods}

Patients and samples. A total of 24 cervical cancer tissue specimens, 24 matched peri-carcinomatous tissue specimens and 16 normal cervical tissue specimens were obtained from the First Affiliated Hospital of Xi'an Jiaotong University between January, 2014 and December, 2017. All patients were diagnosed by two senior pathologists, and no patient had received chemotherapy or radiotherapy prior to surgery; details of the patient characteristics are presented in Table I. Each patient enrolled in this study had volunteered and provided written informed consent. This study was approved by the Ethics Committee of the First Affiliated Hospital of Xi'an Jiaotong University (G-272) in Shaanxi, China.

The cervical cancer samples were collected as previous described (23). After the tissues were dissected, each sample was washed with sterilized PBS and stored at $-80^{\circ} \mathrm{C}$. All procedures were performed on ice.

Data mining. Data obtained from the Gene Expression Profiling Interactive Analysis (GEPIA; http://gepia. cancer-pku.cn/) database was utilized to analyze the mRNA expression level of $H A V C R 2 / L G A L S 9$ in cervical cancer and normal cervical tissues. The correlation between HAVCR2 and $L G A L S 9$ expression was determined by Pearson's correlation analysis. In addition, the association between a high and low HAVCR2/LGALS9 mRNA expression with the overall survival (OS) of patients with cervical cancer was analyzed by Kaplan-Meier analysis, and the hazard ratio (HR) and log-rank P-value were also computed.

$H P V-D N A$ testing. HPV-DNA of 24 cervical cancer tissue samples was examined by polymerase chain reaction (PCR) and flow-through hybridization. In total, 21 HPV genotypes were qualitatively examined using the HPV genotyping test kit according to alkaline phosphatase system (HybriBio), including high-risk HPV types, namely HPV16, 18, 31, 33, 35, $39,45,51,52,56,58,59$ and 68 , and common HPV types in China, namely HPV53, 66 and CP8304.

Cell lines, culture conditions, overexpression and construction of cell lines with gene silencing. The two human cervical cancer cell lines, HeLa and C33A, were obtained from the Cell Bank, Shanghai Institutes for Biological Sciences, Chinese Academy of Sciences, Shanghai. All these cells were cultured in high-glucose Dulbecco's modified Eagle's medium (DMEM; HyClone) supplemented with $10 \%$ fetal bovine serum (FBS; Biological Industries) at $37^{\circ} \mathrm{C}$ in an atmosphere of $5 \% \mathrm{CO}_{2}$. For the preparation of Plenti-CMV-puro-Dest vector containing the HPV18 E6/E7/E67 fragment, the HPV18 E6/E7/E67 fragment was cloned from the genomic DNA of the HPV18(+) cell line, HeLa. The DNA fragment was treated with Kpn1 and $P s t 1$, and the target gene was then ligated into the entry vector pENTR-MCS. The plasmid Plenti-CMV-puro-Dest and pENTR-MCS recombination reactions were performed using LR Clonase II (Invitrogen; Thermo Fisher Scientific). Lipofectamine 2000 (Invitrogen; Thermo Fisher Scientific) was used to transfect the plasmid into the HPV(-) C33A cell line. The transduced cells were then selected with puromycin. Stably transduced cells were maintained in culture in the presence of puromycin. The cell lines were named C33A-18E6, C33A-18E7, and C33A-18E6/E7. The expression of the genes was determined by RT-qPCR.

The HeLa tumor cells were transfected with scramble, HPV18-E6, HPV18-E7, EZH2 and DNMT3A-specific siRNA (GenePharma), the following siRNA oligos for EZH2, HPV18-E6, HPV18-E7 and DNMT3A are listed in Table II. The siRNAs were transfected using X-tremeGENE siRNA Transfection Reagent (Roche), and the transfected cells were analyzed for HPV18-E6, HPV18-E7, EZH2 and DNMT3A expression levels by RT-qPCR and western blot analysis. All cell lines were named 18E6-siRNA, 18E7-siRNA, 18E6/E7-siRNA, EZH2-siRNA and DNMT3A-siRNA.

5-Aza-2'-deoxycytidine (5-Aza-CdR) treatment. A total of $1.0 \times 10^{5} \mathrm{HeLa}$ and C33A cells per well were cultured separately in 6-well plates in DMEM with 10\% FBS. After $24 \mathrm{~h}$, the medium was replaced with fresh medium containing 0 , 2.5 or $5 \mu \mathrm{M}$ 5-Aza-CdR (Sigma-Aldrich; Merck KGaA). The medium containing 5-Aza-CdR was replaced every $24 \mathrm{~h}$ during a 72-h period, as previously described (23).

DNA extraction, bisulfite modification and methylationspecific PCR (MS-PCR). Genomic DNA was isolated from the cells and tissues using a Takara Mini BEST Universal Genomic DNA Extraction kit (Takara) according to the manufacturer's instructions. DNA modification was performed as previously described (23). A total of $500 \mathrm{ng}$ of the extracted DNA was bisulfite-modified with the EZ DNA Methylation-Gold ${ }^{\mathrm{TM}}$ kit (Zymo Research). Modified DNA templates were used for MS-PCR with Zymo TaqTM PreMix (E2003; Zymo Research) following the instructions of the manufacturer. The online software MethPrimer (http://www.urogene.org/methprimer/) profiled $\mathrm{CpG}$ islands in the region that is located from 
Table I. Patient characteristics $(n=24)$.

\begin{tabular}{lr}
\hline Item & No. \\
\hline Age (years) & \\
$\leq 44$ & 10 \\
$>44$ & 14 \\
Clinical stages & \\
Ia & 0 \\
Ib & 8 \\
IIa & 11 \\
IIb & 5 \\
Pathological pattern & \\
Squamous cell carcinoma & 21 \\
Adenocarcinoma & 3 \\
Pathological grading & 1 \\
I & 19 \\
II & 4 \\
III & \\
Lymph nodes metastasis & 3 \\
Yes & 21 \\
No & \\
HPV infection & 16 \\
Positive & \\
Negative & \\
\hline
\end{tabular}

$-2,000$ to -200 bp upstream of ATG in the HAVCR2/LGALS9 promoters. One pair of primers was designed to amplify the HAVCR2/LGALS 9 promoter regions. The primer pairs used for MS-PCR are listed in Table II. The thermocycling conditions were as follows: $95^{\circ} \mathrm{C}$ for $10 \mathrm{~min}$ and 40 cycles of $95^{\circ} \mathrm{C}$ for $30 \mathrm{sec}$. The annealing temperature for the methylated primer pairs of $H A V C R 2$ and $L G A L S 9$ was $60^{\circ} \mathrm{C}$, respectively, while that for the unmethylated primer pairs was $55^{\circ} \mathrm{C}$ and $56.3^{\circ} \mathrm{C}$, respectively for $30 \mathrm{sec}$ and $72^{\circ} \mathrm{C}$ for $30 \mathrm{sec}$, followed by an incubation step at $72^{\circ} \mathrm{C}$ for $7 \mathrm{~min}$. The MS-PCR products were separated on a $2 \%$ agarose gel, stained with Gelview and visualized under ultraviolet illumination (Bio-Rad). Methylation level was calculated by the ratio of methylated and unmethylated levels. The calculation method was as follows: Methylation $(\mathrm{M})=\mathrm{M} /(\mathrm{M}+\mathrm{U})$; unmethylation $=\mathrm{U} /(\mathrm{M}+\mathrm{U})$. The grey value of each band represented its relative expression and was measured using Image J software. Each reaction was performed in triplicate.

RNA extraction and reverse transcription-quantitative $P C R(R T-q P C R)$. Total RNA was extracted from cells using TRIzol Reagent (Life Technologies; Thermo Fisher Scientific) according to the manufacturer's instructions and as previously described (24). The RNA was then subjected to reverse transcription with a cDNA PrimeScript ${ }^{\mathrm{TM}}$ RT Master Mix (Takara). The primers used for qPCR are listed in Table II. qPCR was carried out using SYBR-Green II Premix (Takara) by a two-step amplification procedure according to the manufacturer's protocol. The reaction conditions were as follows: $95^{\circ} \mathrm{C}$ for $30 \mathrm{sec}, 40$ cycles of $95^{\circ} \mathrm{C}$ for $5 \mathrm{sec}$ and $60^{\circ} \mathrm{C}$ for $30 \mathrm{sec}, 72^{\circ} \mathrm{C}$ for $5 \mathrm{sec}$. We used the cycle threshold (CT) as the representative point. $G A P D H$ was used as anternal reference gene. The relative expression of HPV18 E6 and E7 in each group (fold change compared with the control) was calculated using the formula: $R Q=2^{-\Delta \Delta C q}(25)$. Each reaction was performed in triplicate.

Western blot analysis. The cells were harvested in RIPA lysis buffer containing $1 \mathrm{mM}$ protease inhibitor cocktail and $1 \mathrm{mM}$ PMSF. The protein determination was assessed using a BCA detection kit (Beyotime Biotechnology, China). Proteins were resolved by $12 \%$ SDS-PAGE and electroblotted onto a polyvinylidene fluoride membrane (Millipore), $40 \mu \mathrm{g}$ of protein loaded per lane, which was blocked for $1 \mathrm{~h}$ at room temperature in 5\% skim milk, $1 \mathrm{X}$ TBS, $0.1 \%$ Tween-20. After blocking, the membrane was incubated with primary antibodies overnight at $4^{\circ} \mathrm{C}$ followed by incubation with HRP-conjugated secondary antibodies for $1 \mathrm{~h}$ at room temperature. The antibodies used were as follows: Rabbit polyclonal antibody against human Tim-3 (1:500 dilution, ab185703; Abcam), rabbit polyclonal antibody against human galectin-9 (1:100 dilution, ab123712; Abcam), mouse monoclonal antibody against human DNMT3A (1:200 dilution, sc-365769; Santa Cruz Biotechnology), rabbit monoclonal antibody against human EZH2 (1:1,000 dilution, 5246; Cell Signaling Technology), rabbit monoclonal antibody against human H3K27me3 (1:1,000 dilution, 9733; Cell Signaling Technology), mouse monoclonal antibody against HPV18 E6 (1:250 dilution, NB100-2729; Novus Biologicals) and mouse monoclonal antibody against HPV18 E7 (1:250 dilution, NB110-17215; Novus Biologicals), $\beta$-actin mouse monoclonal antibody (1:500 dilution, 60008-1-lg; Proteintech). The secondary antibodies were as follows: HRP-conjugated rabbit anti-mouse IgG (1:5,000 dilution, D110273-0100; BBI Life Sciences), HRP-conjugated goat anti-rabbit IgG (1:5,000 dilution, D110058; BBI Life Sciences). All antibodies were diluted by $1 \mathrm{X}$ TBST. The chemiluminescence signal was detected following incubation with enhanced chemiluminescence reagent (Millipore, Mass). The grey value of each band was measured with ImageJ software $(1.47 \mathrm{v})$.

Chromatin immunoprecipitation (ChIP) assay and ChIP-qPCR. ChIP assays were carried out using the Simple ChIP Enzymatic Chromatin IP kit (9003; Cell Signaling Technology). Anti-H3K27me3 antibody (1:50 dilution, 9733; Cell Signaling Technology), anti-EZH2 antibody (1:100 dilution, 5246; Cell Signaling Technology), anti-DNMT3A antibody (1:50 dilution, ab2850; Abcam), anti-E2F-1 antibody (1:100 dilution, 3742; Cell Signaling Technology), anti-histone H3 antibody (1:50 dilution, 4620; Cell Signaling Technology), anti-IgG antibody (1:1,000 dilution, 2729; Cell Signaling Technology) and anti-FOXM1 antibody (1:100 dilution, 20459; Cell Signaling Technology) were used according to the manufacturer's instructions. The antibodies were incubated overnight at $4^{\circ} \mathrm{C}$. The DNMT3A, EZH2 and $H A V C R 2 / L G A L S 9$ promoters DNA were detected by RT-qPCR using the promoter DNA-specific primers listed in Table II. RT-qPCR was performed as described above. IgG was used as a negative control and histone $\mathrm{H} 3$ was used as a positive control. 
Table II. Primer sequences.

\begin{tabular}{|c|c|c|}
\hline Name & Application & Sequence \\
\hline DNMT3A-ChIP-F1 & ChIP-qPCR & ATCATCAGTAGGGCGGGGTGGCCAC \\
\hline DNMT3A-ChIP-R1 & ChIP-qPCR & CTCCAATGCTTCCAGGTCCCTCCGT \\
\hline DNMT3A-ChIP-F2 & ChIP-qPCR & TTGGAGAACCTCCCGAAGGAAAACC \\
\hline DNMT3A-ChIP-R2 & ChIP-qPCR & GCCACCCTTTTAGCGTCACAGAACC \\
\hline DNMT3A-ChIP-F3 & ChIP-qPCR & CGTTGGGGGGGCGGGTGCTGGGCTG \\
\hline DNMT3A-ChIP-R3 & ChIP-qPCR & TGACTGGCACAGGACATGGCGTGCT \\
\hline DNMT3A-ChIP-F4 & ChIP-qPCR & CATGGGGAAGGAGAACAGCCCCCAC \\
\hline DNMT3A-ChIP-R4 & ChIP-qPCR & GCACTGGAAGACTGAAAGATTTCAT \\
\hline EZH2-ChIP-F1 & ChIP-qPCR & GCTCACGCCTGTAATCCCAGCACTT \\
\hline EZH2-ChIP-R1 & ChIP-qPCR & GGAGTTTCGCTCTGGTTGTCCAGGC \\
\hline EZH2-ChIP-F2 & ChIP-qPCR & GGCTGAGGCATGAGAATCGCTTGAA \\
\hline EZH2-ChIP-R2 & ChIP-qPCR & TGAGACGGAGTTTCGCTCTGGTTGT \\
\hline EZH2-ChIP-F3 & ChIP-qPCR & GCCTGCACACCGCCTTCCTGAGAGG \\
\hline EZH2-ChIP-R3 & ChIP-qPCR & GGGGTTCGCTGTAAGGGACGCCACT \\
\hline EZH2-ChIP-F4 & ChIP-qPCR & CCACACGGCCAGTGGCGTCCCTTAC \\
\hline EZH2-ChIP-R4 & ChIP-qPCR & CACGCAGAGTGCGCTCAGGGCTCGT \\
\hline HAVCR2-ChIP-F1 & ChIP-qPCR & GTGGAAAAAATCTGTCACTTAGGGG \\
\hline HAVCR2-ChIP-R1 & ChIP-qPCR & ATTTTTAGTAGAGACGGGGTTTCTC \\
\hline HAVCR2-ChIP-F2 & ChIP-qPCR & ССТGTAATCCCAGCTACTCAGGAGG \\
\hline HAVCR2-ChIP-R2 & ChIP-qPCR & CTTGTTCAATGTGTGTACTTCCCAT \\
\hline HAVCR2-ChIP-F3 & ChIP-qPCR & CCCAATGCATTTAATGGCATAAATG \\
\hline HAVCR2-ChIP-R3 & ChIP-qPCR & CAGCCACACTCCCATAACTGAGGTA \\
\hline HAVCR2-ChIP-F4 & ChIP-qPCR & GGAACTCAACACTTTCTGATCATTC \\
\hline HAVCR2-ChIP-R4 & ChIP-qPCR & GACTTTGACCTTCAAACTTCCAACT \\
\hline LGALS9-ChIP-F1 & ChIP-qPCR & GGTAGAGTAAAATGTACAGATCCTG \\
\hline LGALS9-ChIP-R1 & ChIP-qPCR & GCGAGACCTTGTCTCTACTAAAAAT \\
\hline LGALS9-ChIP-F2 & ChIP-qPCR & TCAGCCTCCCAATGTGCTGAATTAC \\
\hline$L G A L S 9-C h I P-R 2$ & ChIP-qPCR & CCAGATCCAAACTTGACTTGAAGTG \\
\hline LGALS9-ChIP-F3 & ChIP-qPCR & TCCTGTGGCCTAGCTCCTTTTTATT \\
\hline LGALS9-ChIP-R3 & ChIP-qPCR & AGAAAAACTGCTTGGTGAGTTGTAA \\
\hline LGALS9-ChIP-F4 & ChIP-qPCR & CACATATGTTTTCСTTTCTCTTGGG \\
\hline LGALS9-ChIP-R4 & ChIP-qPCR & ACACCTGTGGTCTCAGCTACATGGG \\
\hline HPV18-E6-F & RT-qPCR & CAACACGGCGACCCTACAAG \\
\hline HPV18-E6-R & RT-qPCR & GCTGGATTCAACGGTTTCTGG \\
\hline HPV18-E7-F & RT-qPCR & ACATTTACCAGCCCGACG \\
\hline HPV18-E7-R & RT-qPCR & CAAAGGACAGGGTGTTCAGA \\
\hline$G A P D H-\mathrm{F}$ & RT-qPCR & GCACCGTCAAGGCTGAGAAC \\
\hline$G A P D H-\mathrm{R}$ & RT-qPCR & TGGTGAAGACGCCAGTGGA \\
\hline$H A V C R 2-\mathrm{ML}$ & MS-PCR & TATAAAATGAGAAATTGGTCGGGCG \\
\hline$H A V C R 2-\mathrm{MR}$ & MS-PCR & TTACAAACATATACCACCACCCCGA \\
\hline HAVCR2-UL & MS-PCR & GAAATTGGTTGGGTGTGGTGGTTAT \\
\hline$H A V C R 2-\mathrm{UR}$ & MS-PCR & TATACCACCACCCCAAATAATTTTA \\
\hline$L G A L S 9-\mathrm{ML}$ & MS-PCR & TTTTCGAGATAGGTTTGCGATTTTG \\
\hline$L G A L S 9-\mathrm{MR}$ & MS-PCR & AATACCGACACCCTTCAATCACCAC \\
\hline LGALS9-UL & MS-PCR & GAGTTTTTGAGATAGGTTTGTGATT \\
\hline LGALS9-UR & MS-PCR & ATACCAАCACCСТTCAАTCACCACA \\
\hline EZH2-sense & Gene silencing & CGGCUUCCCAAUAACAGUATT \\
\hline$E Z H 2$-anti-sense & Gene silencing & UACUGUUAUUGGGAAGCCGTT \\
\hline$D N M T 3 A$-sense & Gene silencing & GCCAAGGUCAUUGCAGGAATT \\
\hline$D N M T 3 A$-anti-sense & Gene silencing & UUCCUGCAAUGACCUUGGCTT \\
\hline HPV18-E6-sense & Gene silencing & CGCAGAGAAACACAAGUAUTT \\
\hline HPV18-E6-anti-sense & Gene silencing & AUACUUGUGUUUCUCUGCGTT \\
\hline
\end{tabular}


Table II. Continued.

\begin{tabular}{llc}
\hline Name & Application & Sequence \\
\hline HPV18-E7-sense & Gene silencing & GUCACACAAUGUUGUGUAUTT \\
HPV18-E7-anti-sense & Gene silencing & AUACACAACAUUGUGUGACTT \\
Negative control-sense & Gene silencing & UUCUCCGAACGUGUCACGUTT \\
Negative control-sense & Gene silencing & ACGUGACACGUUCGGAGAATT \\
\hline
\end{tabular}

ChIP, chromatin immunoprecipitation; RT-qPCR, reverse transcription-quantitative PCR; MS-PCR, methylation-specific-polymerase chain reaction; F, Forward primer; R, reverse primer; ML/UL, methylation/unmethylation forward primer; MR/UR, methylation/unmethylation backward primer.

Co-immunoprecipitation (co-IP) assays. Immunoprecipitation was carried out to assess the interaction between EZH2, DNMT3A and HPV18 E6 and E7. After harvesting the total protein from the HeLa cells, the supernatants were incubated overnight at $4^{\circ} \mathrm{C}$ with rabbit anti-EZH2 (1:300 dilution, 5246; Cell Signaling Technology) and rabbit anti-DNMT3A (1:50 dilution, ab13888; Abcam) and protein G-Dynabeads (Thermo Fisher Scientific) were then conjugated to EZH2 and DNMT3A. The samples were then electrophoresed through gradient $12 \%$ SDS-polyacrylamide gels and transferred to membranes that were probed with mouse anti-HPV18 E6 and E7 antibodies, respectively (1:250 dilution; Novus Biologicals, NB100-2729/NB110-17215) and antibodies were incubated overnight at $4{ }^{\circ} \mathrm{C}$. This was followed by incubation with horseradish peroxidase-conjugated secondary antibodies for $1 \mathrm{~h}$ at room temperature. The secondary antibodies: HRP-conjugated rabbit anti-mouse $\operatorname{IgG}$ (1:5,000 dilution, D110273-0100; BBI Life Sciences), HRP-conjugated goat anti-rabbit IgG (1:5,000 dilution, D110058; BBI Life Sciences). All antibodies were diluted using 1X TBST. Western blot analysis was performed as described above.

Statistical analysis. Statistical analyses were performed using GraphPad Prism 7 software (GraphPad Software). A paired t-test and one-way ANOVA were carried out on samples within groups, Dunnett's test was used as the post hoc test after one-way ANOVA. A P-value $<0.05$ was considered to indicate a statistically significant difference. The data are presented as the means \pm standard error of the mean (SEM). All experiments were independently repeated at least thrice, with consistent results.

\section{Results}

Protein expressionofTim-3/galectin-9 is increased incervical cancer tissues, and the gene methylation level is decreased. The protein expression of Tim-3/galectin- 9 in the 24 cervical cancer tissues, 24 matched pericarcinomatous tissues and 16 normal cervical tissues was detected by western blot analysis. Compared to that in normal cervical tissues, the average relative expression of Tim-3/galectin-9 was higher in the cervical cancer and pericarcinomatous tissues, although no significant difference was observed between the tumor tissues and pericarcinomatous tissues (Fig. 1A-C). The relative protein expression of Tim-3/galectin-9 in the HPV-positive cervical cancer tissues was higher than that in the HPV-negative cervical cancer tissues (Fig. 1D). Moreover, Tim-3 is encoded by HAVCR2, and galectin-9 is encoded by LGALS9. Using the GEPIA database (http://gepia.cancer-pku.cn/), we compared the mRNA expression of HAVCR2/LGALS9 between the cervical cancer and normal cervical samples. The results indicated that the average mRNA expression levels of HAVCR2 and LGALS9 were higher in the cancer than in the normal cervical samples (Fig. 1E). We then investigated the effects of $H A V C R 2 / L G A L S 9$ on patient prognosis using the GEPIA database. In particular, an increased $L G A L S 9$ expression was associated with a poor overall survival (OS). Of note, no significant association was observed between the expression of $H A V C R 2$ and patient prognosis by the GEPIA database (Fig. 1F). Furthermore, HAVCR2 positively correlated with LGALS9 (r=0.26, $\mathrm{P}<0.05)$ (Fig. 1G). In addition, the results of immunohistochemical analysis revealed that Tim-3 and galectin-9 were expressed in the tumor cells of the cervical cancer tissues (Fig. 1H). Furthermore, Tim-3 and galectin-9 were expressed in the HeLa and C33A cells (Fig. 1I).

Further experiments revealed that the HAVCR2/LGALS9 promoters in 9 cervical cancer tissues and 9 normal cervical tissues displayed a hypermethylated status in normal cervical tissues compared with cervical cancer tissue samples, possibly leading to the inhibition of gene expression (Fig. 1J and $\mathrm{K}$ ). Taken together, these results suggest that Tim-3/galectin-9 expression is increased in cervical cancer tissues.

Methylation status in the promoter regions of HAVCR2/LGALS9 in cervical cancer cell lines. The online software, MethPrimer, profiled $\mathrm{CpG}$ islands in the region that is located from $-2,000$ to $-200 \mathrm{bp}$ upstream of ATG and the transcription starts site (TSS) in the HAVCR2/LGALS9 promoters (Fig. 2A). One pair of primers was designed to amplify the $H A V C R 2 / L G A L S 9$ promoter regions. MS-PCR analysis revealed that these regions upstream of ATG were partially methylated in the HeLa and C33A cells (Fig. 2B).

The mRNA expression level of HAVCR2/LGALS9 in the HeLa and C33A cell lines following treatment with the DNA-demethylating reagent, 5-Aza-CdR, was determined to identify whether the methylation status in the promoter regions regulates the expression of $H A V C R 2 / L G A L S 9$ genes at the transcriptional level. The results suggested that the mRNA expression of HAVCR2/LGALS9 in the HeLa and C33A cells increased in a dose-dependent manner following cellular DNA demethylation (Fig. 2C and D). These findings illustrate that 

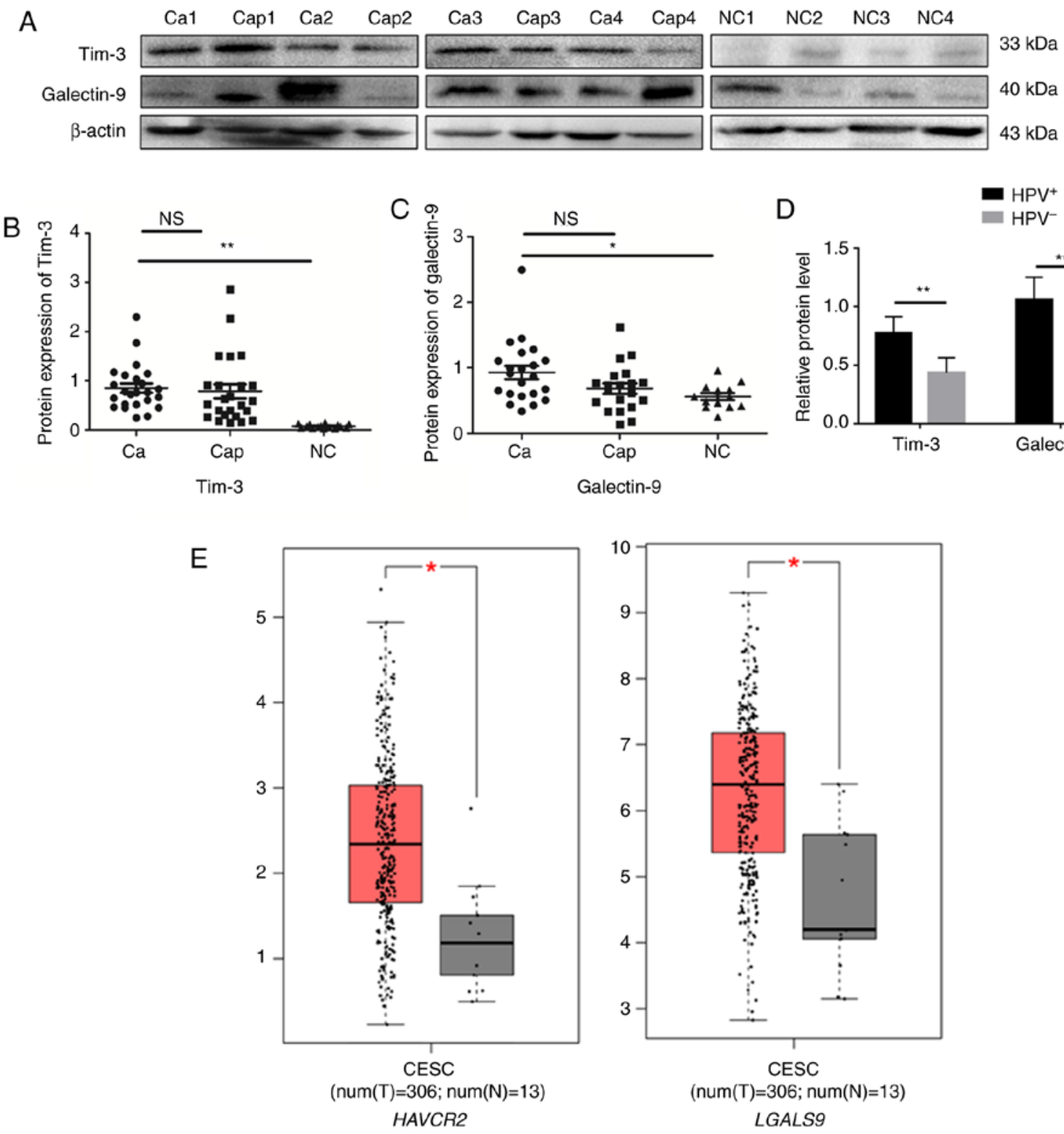

Figure 1. Tim-3/galectin-9 protein and gene methylation levels in human cervical cancer tissues. (A) The protein levels of Tim-3 and galectin-9 in cervical cancer $(\mathrm{Ca})$, para-carcinoma (Cap) $(\mathrm{n}=24)$ and normal cervical tissues $(\mathrm{NC})(\mathrm{n}=16)$ detected by western blot analysis. Blot images of 4 representative samples are shown from each group. (B) Densitometric analysis of Tim-3 and (C) galectin-9 protein levels in cervical cancer $(\mathrm{n}=24)$, para-carcinoma $(\mathrm{n}=24)$ and normal cervical tissues ( $\mathrm{n}=16)$. (D) Gray level analysis of Tim-3/galectin-9 protein expression in HPV-positive $(\mathrm{n}=16)$ and -negative $(\mathrm{n}=8)$ cervical cancer tissues (E) The mRNA expression level of HAVCR2/LGALS9 in cervical cancer, analyzed by GEPIA. (M, methylated; U, unmethylated); methylated and unmethylated levels were quantified as $\mathrm{M} / \mathrm{M}+\mathrm{U} \%$ and $\mathrm{U} / \mathrm{M}+\mathrm{U} \%$, respectively. ${ }^{*} \mathrm{P}<0.05 ;{ }^{* *} \mathrm{P}<0.01$; ns, not significant.

HAVCR2/LGALS9 expression was reversed by 5 -Aza-CdR, which promoted the expression of the HAVCR2/LGALS9 genes at the transcriptional level.

Methylationlevelsin the promoter regions of HAVCR2/LGALS9 are regulated by DNMT3A in cervical cancer cell lines. Following the knockdown of DNMT3A by siRNA, the expression level of Tim-3/galectin-9 was increased (Fig. 3A and B), and in turn, the degree of methylation of the genes encoding these proteins was decreased (Fig. 3C and D). These results suggest that DNMT3A plays an important role in regulating Tim-3/galectin-9 expression. To confirm this causal association, we performed ChIP analysis to evaluate the mechanisms underlying the DNA methylation-mediated regulation of Tim-3/galectin-9. ChIP analysis revealed the enhanced binding of DNMT3A (Fig. 3F and $\mathrm{H}$ ) to the HAVCR2/LGALS9 promoters (Fig. 3E and G) in the HeLa cells. Taken together, the results indicated that DNMT3A downregulated expression of the negative costimulatory molecules Tim-3/galectin- 9 by upregulating the HAVCR2/LGALS9 methylation levels in cervical cancer.

EZH 2 and H3K27me3 suppress DNMT3A expression in HeLa cells. The protein expression of EZH2, H3K27me3 and DNMT3A was detected by western blot analysis in 24 cervical cancer tissues, 24 matched pericarcinomatous tissues and 16 normal cervical tissues. The average relative expression levels of EZH2 and H3K27me3 were higher in the cervical cancer tissues than the controls, and DNMT3A expression was lower in the cancer tissues (Fig. 4A and B). 
$\mathrm{F}$

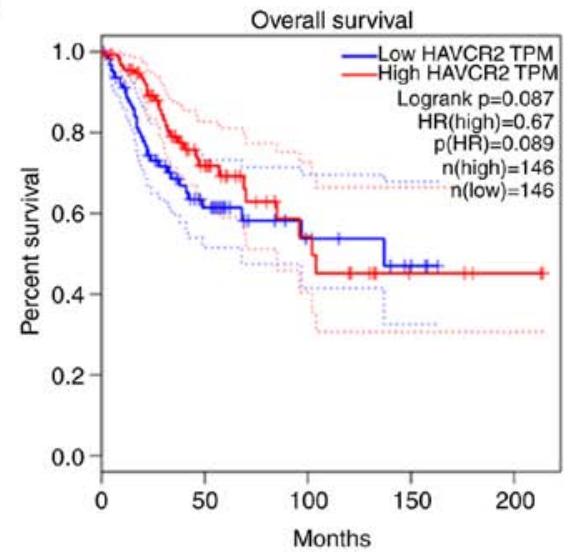

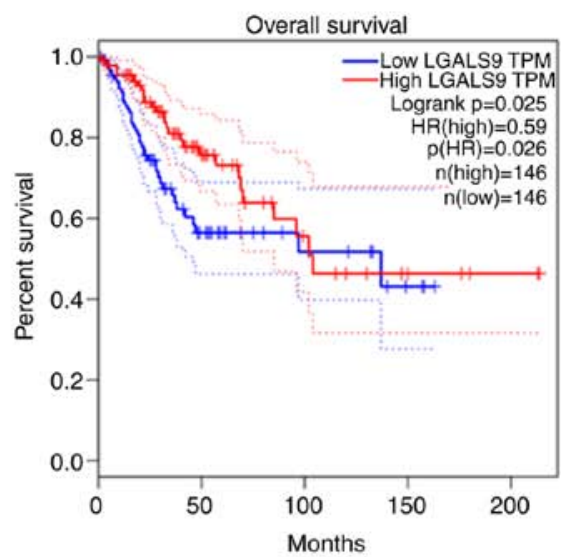
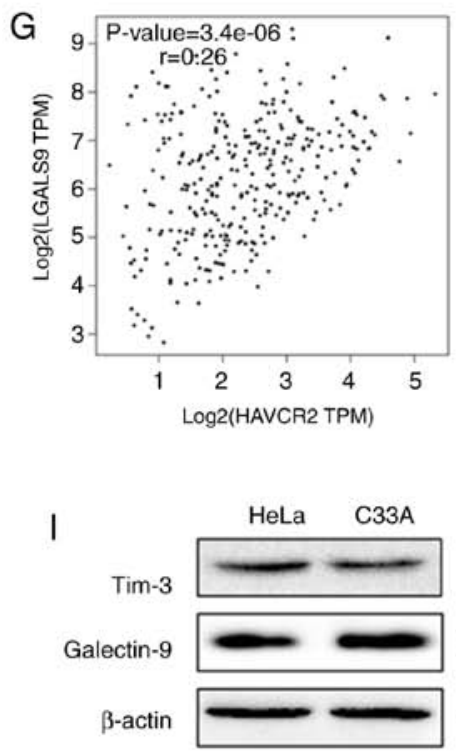

$\mathrm{H}$

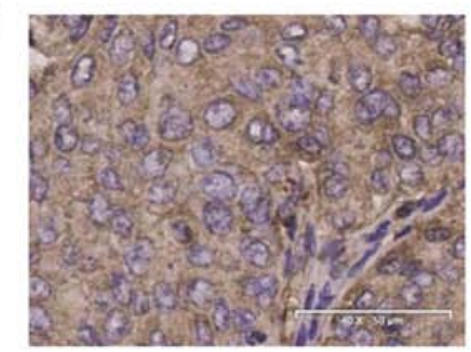

Tim-3

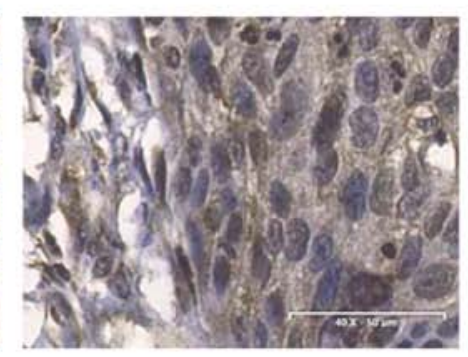

Galectin-9
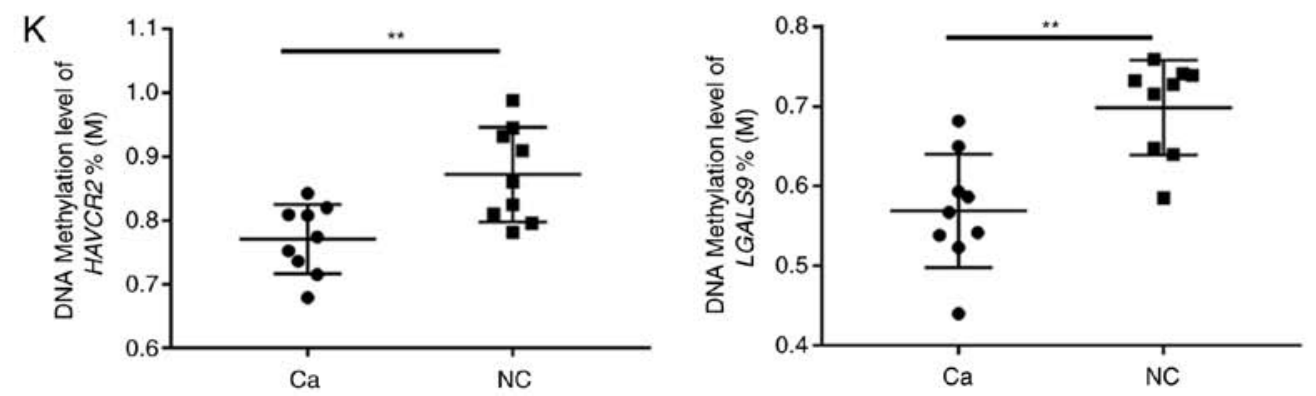

Figure 1. Continued. (F) The prognostic value of mRNA level of HAVCR2/LGALS9 in cervical cancer patients, analyzed by GEPIA. (G) The correlation between HAVCR2/LGALS9 in cervical cancer, analyzed by GEPIA. (H) Positive immunohistochemistry staining of Tim-3 and galectin-9 in representative cervical cancer samples. Magnification, x400. (I) Tim-3 and galectin-9 expression in HeLa and C33A cells detected by western blot analysis. (J) Methylation level of HAVCR2/LGALS9 promoter regions in cervical cancer $(\mathrm{n}=9)$ and normal cervical tissues $(\mathrm{n}=9)$ detected by methylation-specific PCR (MS-PCR). (K) Gray level analysis of HAVCR2/LGALS9 methylation level in cervical cancer and normal cervical tissues. (M, methylated; U, unmethylated); methylated and unmethylated levels were quantified as $\mathrm{M} / \mathrm{M}+\mathrm{U} \%$ and $\mathrm{U} / \mathrm{M}+\mathrm{U} \%$, respectively. ${ }^{*} \mathrm{P}<0.05 ;{ }^{* * *} \mathrm{P}<0.01$; ns, not significant.

H3K27me3 expression was downregulated and DNMT3A expression was upregulated when EZH2 was knocked down by siRNA in the HeLa cells (Fig. 4C). Moreover, the methylation levels of the HAVCR2/LGALS9 genes were upregulated (Fig. 4D and E), and the expression of Tim-3/galectin-9 was downregulated (Fig. 4F). H3K27me3 can thus regulate the methylation level of $H A V C R 2 / L G A L S 9$ by altering the expression of DNMT3A.
EZH2 targets H3K27me3 as part of polycomb repressive complex 2 (PRC2), and its increased expression has been linked to a number of malignancies, with the highest EZH2 protein levels being associated with advanced disease and a poor prognosis (27). In this study, in a screen for epigenetic mechanisms that regulate DNMT3A expression in HeLa cells, ChIP analysis revealed that the HeLa cells exhibited the highest EZH2 and H3K27me3 densities in a region upstream 
A CpG Island
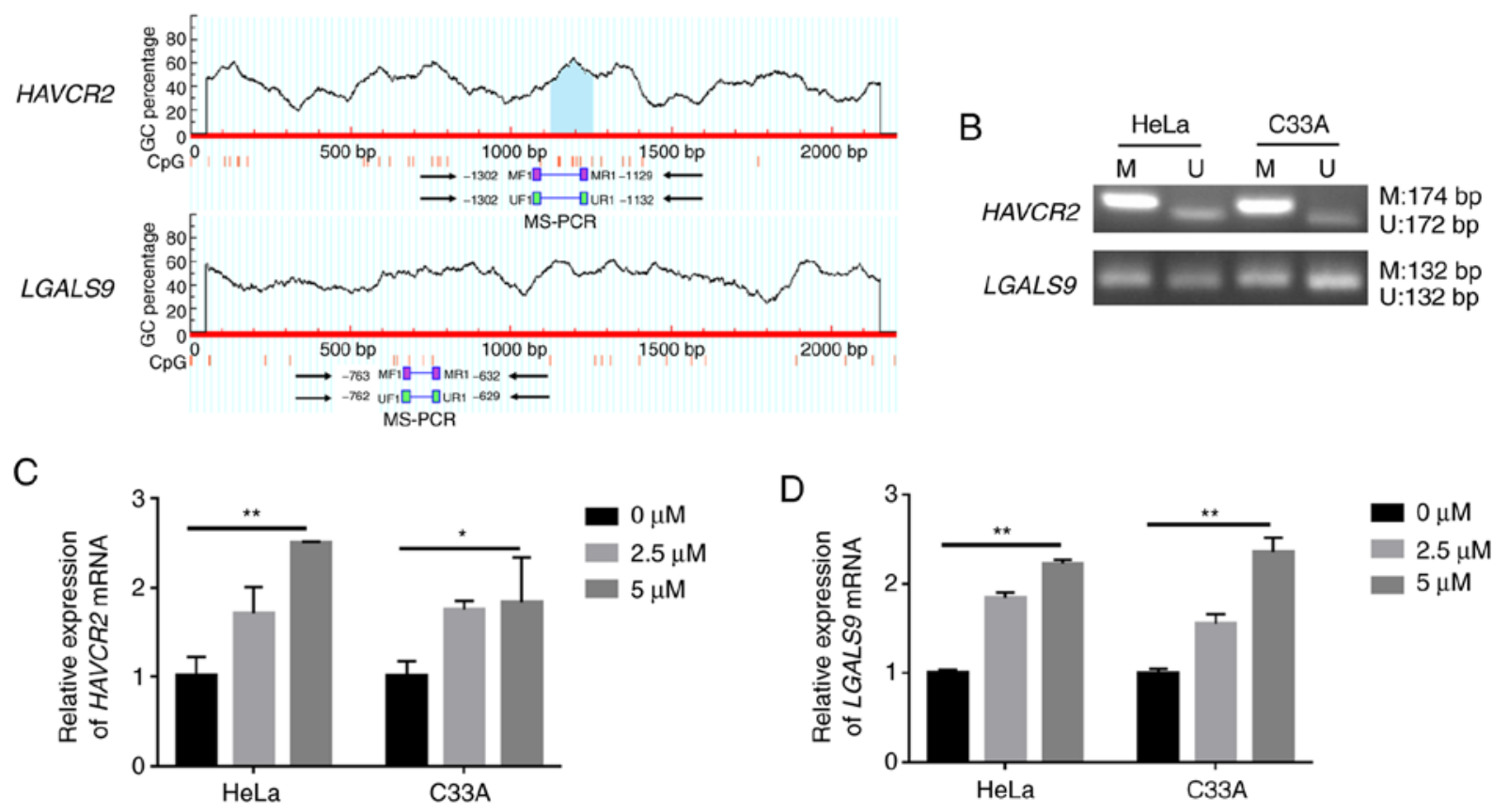

Figure 2. Detection of the methylation status in the promoter regions of HAVCR2/LGALS9 in cervical cancer cell lines. (A) Predicted CpG islands in the promoter region of HAVCR2/LGALS9. Numbers indicate the positions in bp relative to the transcription start site. The blue region represents the CpG islands, and the red vertical bars are the $\mathrm{CpG}$ loci in these input sequences. (B) Detection of HAVCR2/LGALS9 methylation status by methylation-specific PCR (MS-PCR) in HeLa and C33A cell lines; (M, methylated; U, unmethylated). (C) and (D) Relative expression of HAVCR2/LGALS9 mRNA in HeLa and C33A cells following treatment with various concentrations of 5-Aza-CdR. ${ }^{*} \mathrm{P}<0.05$; ${ }^{* *} \mathrm{P}<0.01$. 5-Aza-CdR, 5-aza-2'-deoxycytidine.

of the transcription initiation region (Fig. 4G). EZH2 and H3K27me3 negatively regulated the expression of DNMT3A by acting on the $-1,000$ to +1 region in the promoter of $D N M T 3 A$ (Fig. $4 \mathrm{H}$ and I). These results suggest that $\mathrm{EZH} 2$ and $\mathrm{H} 3 \mathrm{~K} 27 \mathrm{me} 3$ can alter the expression of the negative costimulatory molecules Tim-3/galectin- 9 by synergistically regulating the expression of DNMT3A.

HPV18 oncoproteins E6/E7 can alter the methylation and expression level of Tim-3/galectin-9 in cervical cancer cells. The mRNA expression of HPV18 E6 and E7 was increased following the overexpression of HPV18 E6 and E7 (Fig. 5A). The protein expression of DNMT3A was decreased when HPV18 E6 and/or E7 were overexpressed in the C33A cells (Fig. 5C). At the same time, the knockdown of E6 and/or E7 in the HeLa cells significantly decreased HPV18 E6 and E7 expression (Fig. 5B). In addition, the knockdown of E6 and/or E7 in the HeLa cells increased DNMT3A protein expression (Fig. 5D). These results revealed that HPV18 E6 and/or E7 can lead to a reduction in DNMT3A expression. Co-IP assay also revealed that neither HPV18 E6 nor E7 combined with DNMT3A directly (Fig. 5E).

Furthermore, the data revealed that HPV18 oncoproteins E6 and/or E7 downregulated the methylation level of HAVCR2/LGALS9 significantly (Fig. 5F), and these altered methylation levels contributed to an increased Tim-3/galectin-9 expression among the cell lines with the overexpression of the HPV18 oncoproteins, E6 and/or E7, compared to the C33A cells (Fig. 5H). The knockdown of E6 and/or E7 yielded the opposite results in the HeLa cells (Fig. 5G and I). These results suggest that the HPV18
E6/E7 oncoproteins reduce the methylation level of the $H A V C R 2 / L G A L S 9$ promoters and in turn promote translation of the protein products.

HPV18 oncoproteins E6/E7 participate in the regulation of EZH2 and H3K27me3. EZH2 participates in H3K27me3-mediated gene silencing $(27,28)$. In this study, the $\mathrm{EZH} 2$ and $\mathrm{H} 3 \mathrm{~K} 27 \mathrm{me} 3$ protein levels were strongly decreased when HPV18 oncoproteins were knocked down in the HeLa cells, and the overexpression of HPV18 E6 or/and E7 led to an increase in EZH2 and H3K27me3 expression (Fig. 6A and B). The expression of EZH2 and H3K27me3 in the HeLa cells was higher than that in the $\mathrm{C} 33 \mathrm{~A}$ cells (Fig. 6C). These results suggest that EZH2 and $\mathrm{H} 3 \mathrm{~K} 27 \mathrm{me} 3$ are novel activation targets of HPV18 oncogenes.

However, the expression of HPV oncoproteins E6 or E7 was not altered when EZH2 was knocked down in the HeLa cells (Fig. 6D). Co-IP assays revealed that neither HPV18 E6 nor HPV18 E7 bound EZH2 directly (Fig. 6E). E6 oncoprotein induces FOXM1 expression via the MZF1/NKX2-1 axis in HPV-associated tumorigenesis (29). The viral oncoprotein HPV E7 targets pRb, and the pRb/E2F-1 pathway directly regulates Kv10.1 transcription during G2/M (30-32). E2F-1 is enriched at proximal promoter of EZH2 in MCF-7 cells (33). FOXM1 is enriched on the promoter of EZH2 in HCT116 cells (34). In this study, in a screening for the epigenetic mechanisms that regulated EZH2 expression in HeLa cells, ChIP analysis revealed that the highest FOXM1 and E2F-1 density was found in a region upstream of the transcription initiation region in HeLa cells (Fig. 6F). It has also been shown that FOXM1 and E2F-1 regulates the expression of EZH2 
A

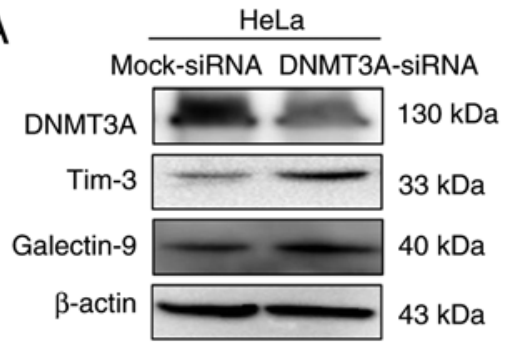

C

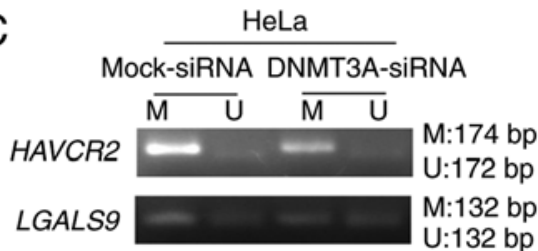

B

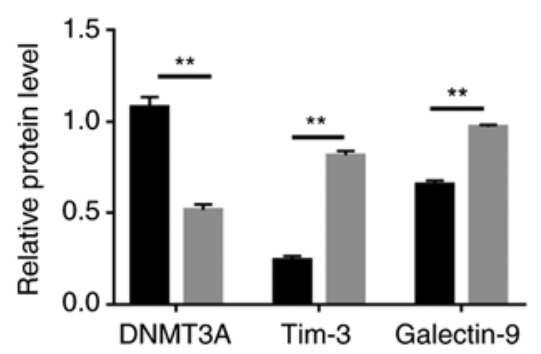

HeLa

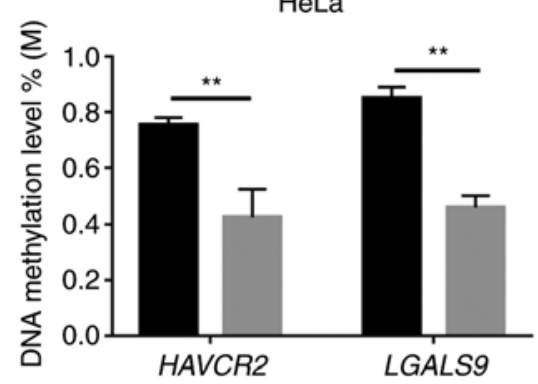

Mock-siRNA

DNMT3A-siRNA

\section{E HAVCR2}

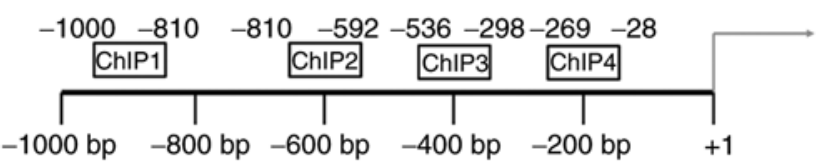

G

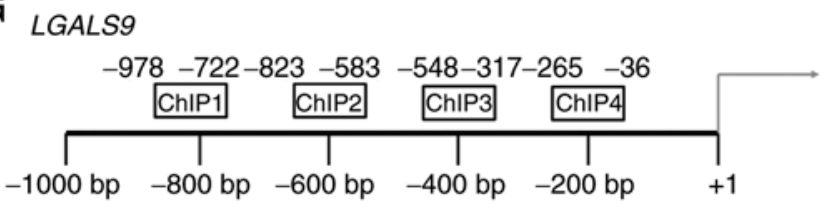

$\mathrm{F}$
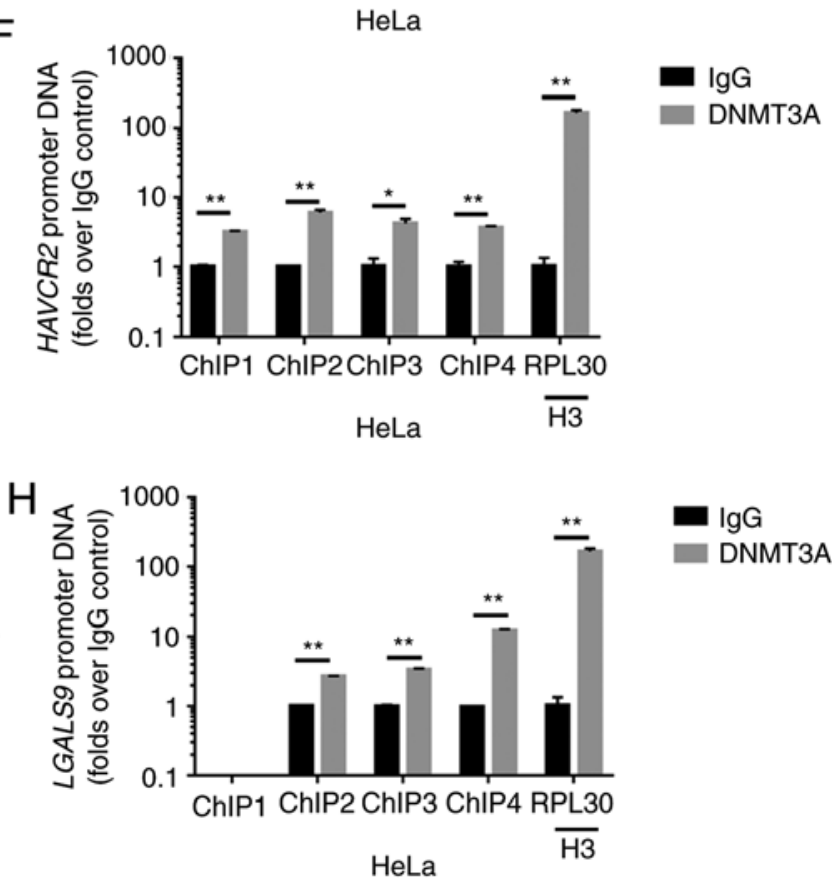

Figure 3. DNMT3A regulates the expression of Tim-3/galectin-9 in cervical cancer cell lines. (A and B) DNMT3A-specific siRNA was transfected into HeLa cells, and the expression of DNMT3A and Tim-3/galectin- 9 was detected by western blot analysis. $\beta$-actin protein was used as a loading control between lanes. (C) MS-PCR analysis was used to examine the methylation level of HAVCR2/LGALS9 in HeLa cells in which DNMT3A was knocked down. (D) Gray level analysis of HAVCR2/LGALS9 methylation levels in HeLa cells in which DNMT3A was knocked down (M, methylated; U, unmethylated); the methylated and unmethylated levels were quantified as $\mathrm{M} / \mathrm{M}+\mathrm{U} \%$ and $\mathrm{U} / \mathrm{M}+\mathrm{U} \%$, respectively. (E and $\mathrm{G}$ ) Schematic representation of the 4 regions of the HAVCR2/LGALSS promoters amplified in the chromatin immunoprecipitation (ChIP)-quantitative PCR (qPCR) experiment. (F and H) Chromatin was cross-linked, fragmented and immunoprecipitated with either IgG (mock) or anti-DNMT3A ChIP-grade antibody and the purified DNA was used to amplify with respective primer pairs for the indicated 4 regions in the HAVCR2/LGALS9 promoters in qPCR. The enrichment of DNMT3A on HAVCR2/LGALS9 promoters relative to IgG in HeLa cells, and $\mathrm{H} 3$ against $R P L 30$ was used as a positive control. ${ }^{*} \mathrm{P}<0.05 ;{ }^{* *} \mathrm{P}<0.01$.

by acting on the $-1,000$ to +1 region of the $E Z H 2$ promoter (Fig. 6G and $\mathrm{H}$ ). Moreover, ChIP analysis did not reveal the enhanced binding of FOXM1 and E2F-1 (Fig. 6I and J) to the DNMT3A promoters in the HeLa cells. Taken together, these findings suggest that HPV18 E6 and E7 promote EZH2 and $\mathrm{H} 3 \mathrm{~K} 27 \mathrm{me} 3$ expression through the interaction of FOXM1 and E2F-1 with the promoter region of EZH2, and inhibit DNMT3A expression.

\section{Discussion}

Epigenetic alterations, such as DNA methylation are essential for the carcinogenesis of cervical cancer, which results in the activation or exclusion of certain genes $(35,36)$. Cicchini et al (37) found that HPV infection distinctly altered the methylation patterns in HPV-associated cancer, and that HPV E7-dependent promoter hypermethylation led to the 

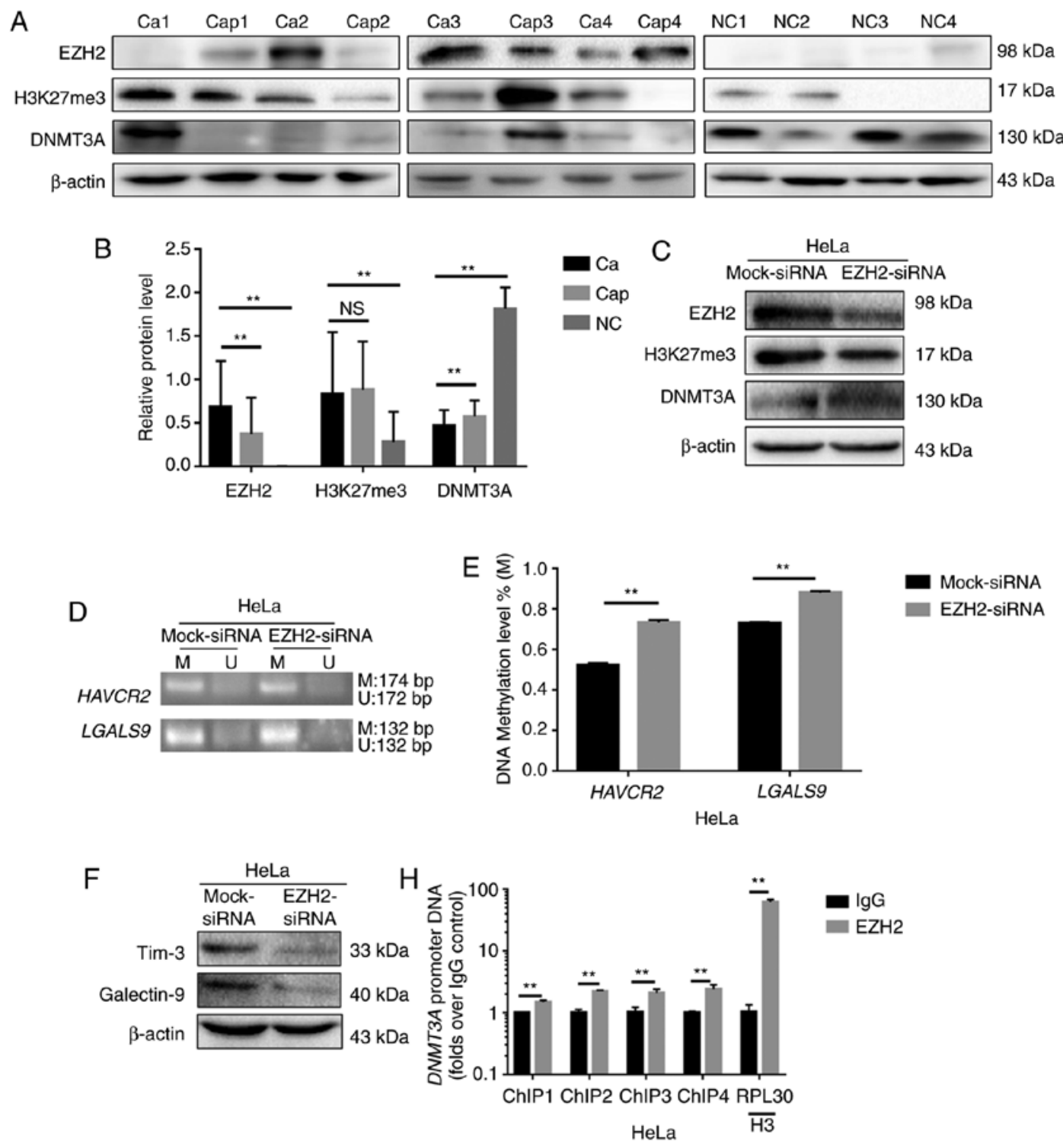

G

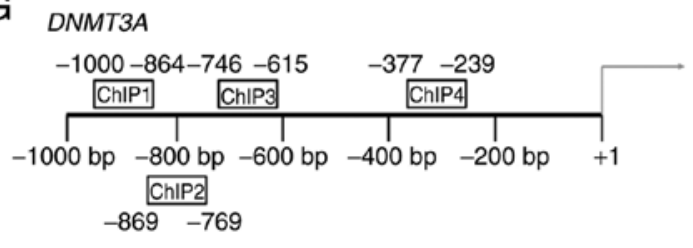

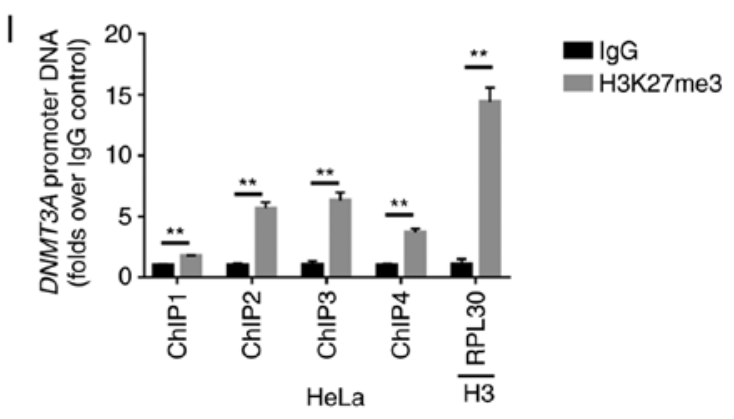

Figure 4. EZH2 and H3K27me3 regulate Tim-3/galectin-9 expression through DNMT3A. (A) The protein levels of EZH2, H3K27me3 and DNMT3A in cervical cancer (Ca), paracarcinoma (Cap) $(n=24)$ and normal cervical tissues $(\mathrm{NC})(\mathrm{n}=16)$ detected by western blot analysis. Blot images of 4 representative samples are shown from each group. (B) Densitometric analysis of EZH2, H3K27me3 and DNMT3A protein levels in cervical cancer ( $\mathrm{n}=24)$, paracarcinoma $(\mathrm{n}=24)$ and normal cervical tissues $(\mathrm{n}=16)$. $(\mathrm{C}$ and $\mathrm{F})$ Western blot analysis of HeLa cells $48 \mathrm{~h}$ following transfection with siRNA against EZH2. $\beta$-actin protein was used as a loading control between lanes. (D) MS-PCR analysis was used to examine the methylation level of HAVCR2/LGALS9. (E) Gray level analysis of HAVCR2/LGALS9 methylation levels in HeLa cells in which EZH2 was knocked down (M, methylated; U, unmethylated); the methylated and unmethylated levels were quantified as $\mathrm{M} / \mathrm{M}+\mathrm{U} \%$ and $\mathrm{U} / \mathrm{M}+\mathrm{U} \%$, respectively. (G) Schematic representation of the 4 regions of the DNMT3A promoter amplified in the chromatin immunoprecipitation (ChIP)-quantitative PCR (qPCR) experiment. (H and I) Chromatin was cross-linked, fragmented and immunoprecipitated with either IgG (mock) or anti-EZH2 and anti-H3K27me3 ChIP-grade antibody and the purified DNA was used to amplify with respective primer pairs for indicated four regions in the DNMT3A promoters in qPCR. The enrichment of EZH2 and H3K27me3 on DNMT3A promoter relative to IgG in HeLa cells, and $\mathrm{H} 3$ against $R P L 30$ was used as a positive control. ${ }^{* *} \mathrm{P}<0.01$; ns, not significant.

downregulation of the chemokine CXCL14 and the suppression of antitumor immune responses. Epigenetically regulated cytokines mediate the expression of tumor-associated genes and manipulate their biological role in cancer (38-40). 
A

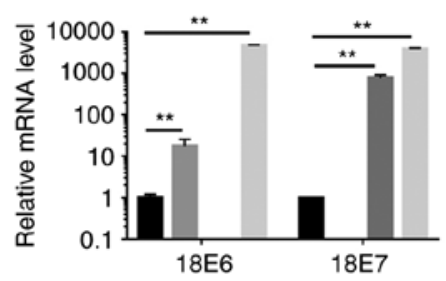

B

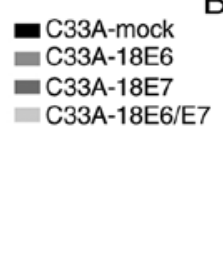

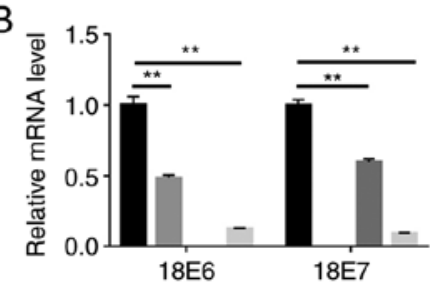

- HeLa-NC

HeLa-siE6

- HeLa-siE7

- HeLa-siE6/E7

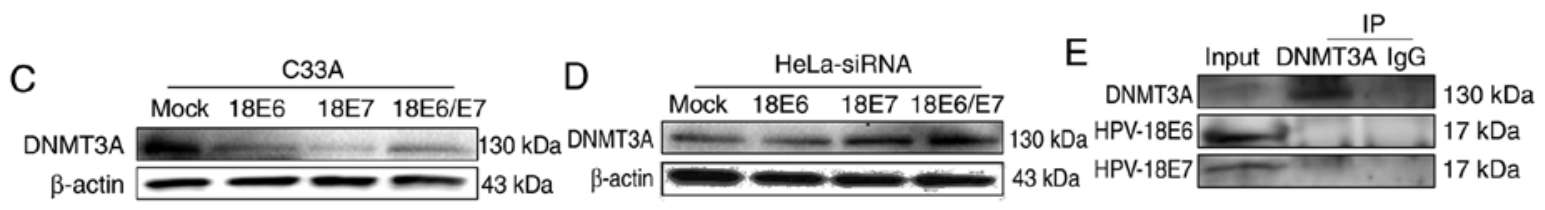
$43 \mathrm{kDa} \quad \beta$-actin

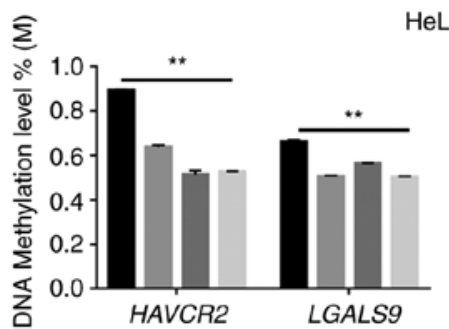

HeLa
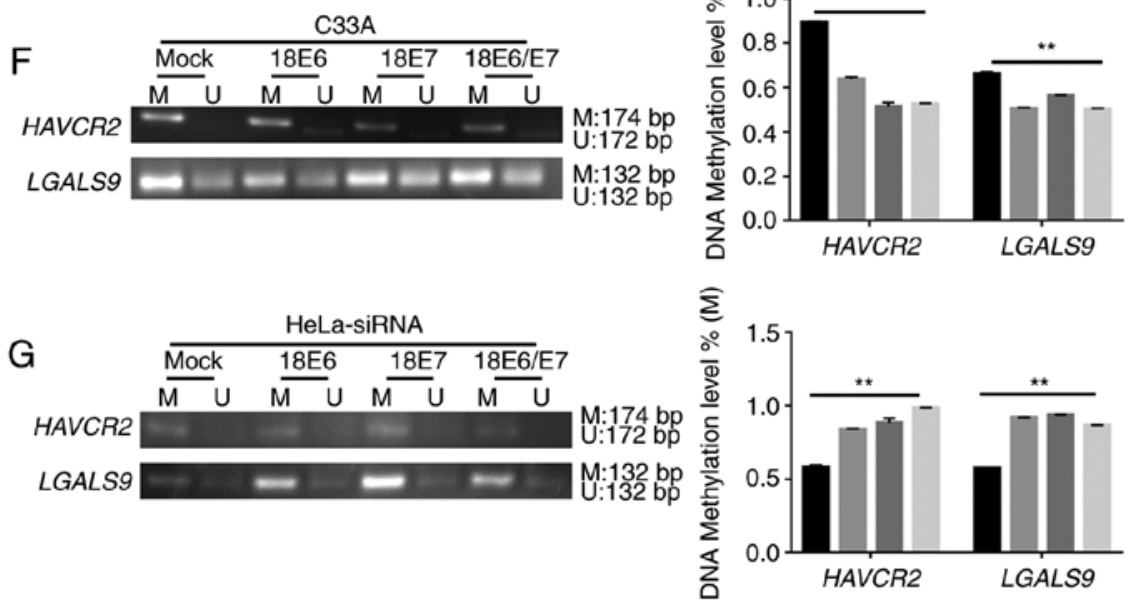

- C33A-mock

-

- C33A-18E7

$17 \mathrm{kDa}$ $17 \mathrm{kDa}$

$\mathrm{H}$

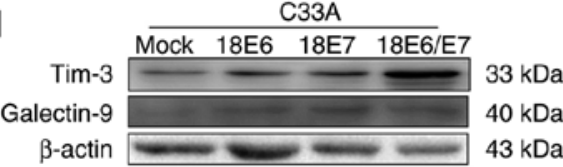

I

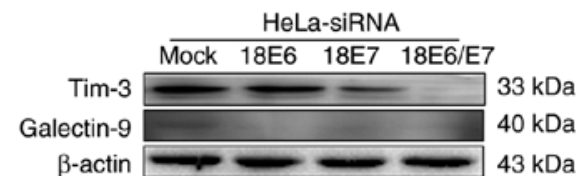

Figure 5. Function of HPV18 oncoproteins E6/E7 in the regulation of Tim-3/galectin-9 and DNMT3A expression in human cervical cancer cells. (A) and (B) mRNA levels in of HPV18 E6/E7-overexpressing C33A cells and of HPV18 E6/E7-knockdown HeLa cells. (C) and (D) C33A cells were transfected with 18E6/E7/E6/7 lentiviral vector, and HeLa cells were transfected with 18E6/E7/E6/7-specific siRNAs. Western blot analysis was then used to detect DNMT3A expression. (E) Co-IP assay was used to analyzed the incorporation between DNMT3A and HPV18 E6/E7. (F and G) The methylation level of the HAVCR2/LGALS9 promoters was monitored by MS-PCR. Gray level analysis of HAVCR2/LGALS9 methylation levels in cervical cancer cells (M, methylated; $\mathrm{U}$, unmethylated); the methylated and unmethylated levels were quantified as $\mathrm{M} / \mathrm{M}+\mathrm{U} \%$ and $\mathrm{U} / \mathrm{M}+\mathrm{U} \%$, respectively. (H and $\mathrm{I}$ ) $\mathrm{C} 33 \mathrm{~A}$ cells were transfected with 18E6/E7/E6/7 lentiviral vector, and HeLa cells were transfected with 18E6/E7/E6/7-specific siRNAs. The expression of Tim-3/galectin-9 was determined by western blot analysis. ${ }^{* *} \mathrm{P}<0.01$.

The Tim-3/galectin-9 pathway, which plays a pivotal role in immune regulation, is differentially regulated in a variety of tumors and is a potential therapeutic target. Tim-3 and galectin-9 have been found to be significantly overexpressed in gastric cancer (41). Additionally, high Tim-3 and low galectin-9 expression levels have been shown to be associated with a poor prognosis of patients with esophageal squamous cell carcinoma (42). A higher expression level of Tim-3 has been shown to be positively associated with a shorter progression-free survival of patients with clear cell renal cell carcinoma (43). Patients with glioma with a high expression of galectin-9 have been shown to exhibit a worse overall survival (44).

In this study, the expression level of Tim-3/galectin-9 was higher in human cervical cancer samples than in normal cervical tissue. The high expression of $L G A L S 9$, but not $H A V C R 2$ was negatively associated with overall survival.
Thus, it can be considered that the increased expression of secreted galectin-9 from cancer cells binds with Tim-3, causing immunosuppression in the tumor microenvironment; this leads to a poor overall survival in cervical cancer patients, suggesting that Tim-3 and galectin-9 promote the occurrence and development of cervical cancer. The levels of Tim-3 and galectin-9 were similar in cancer and para-cancerous tissues; thus, the role of Tim-3 and galectin-9 in paracancerous tissues requires further research, which is a limitation of the present study.

We found that there was partial methylation in the promoter regions of the HAVCR2/LGALS9 genes, which encode negative costimulatory molecules Tim-3/galectin-9 in HeLa and C33A cells and in cervical cancer tissues; these methylation patterns in cancer cells could be reversed by 5 -Aza-CdR treatment, leading to an upregulated Tim-3/galectin-9 expression. 
A

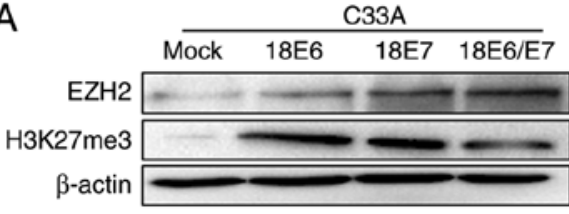

B $98 \mathrm{kDa}$ $17 \mathrm{kDa}$ $43 \mathrm{kDa}$

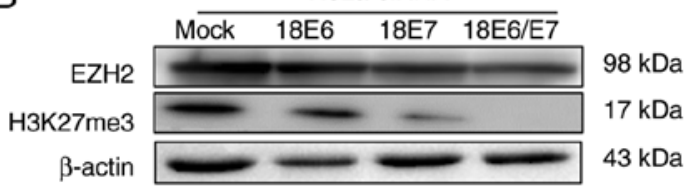

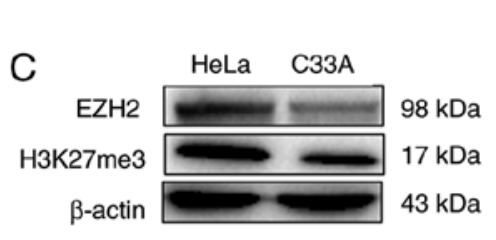

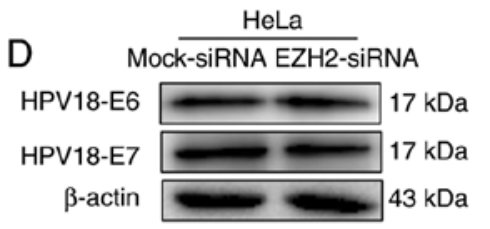

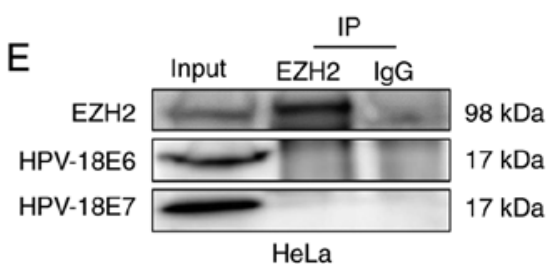

$\mathrm{F}$

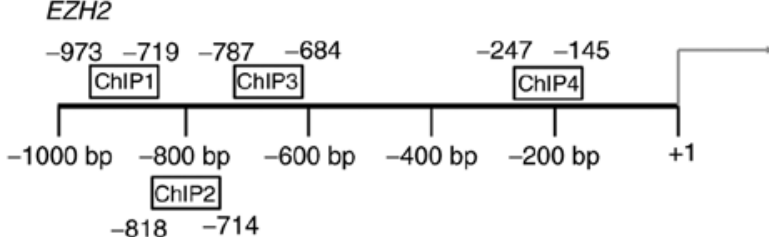

$\mathrm{G}$

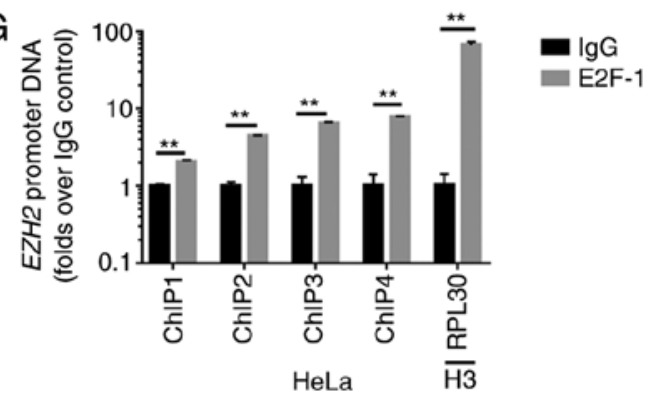

$\mathrm{H}$
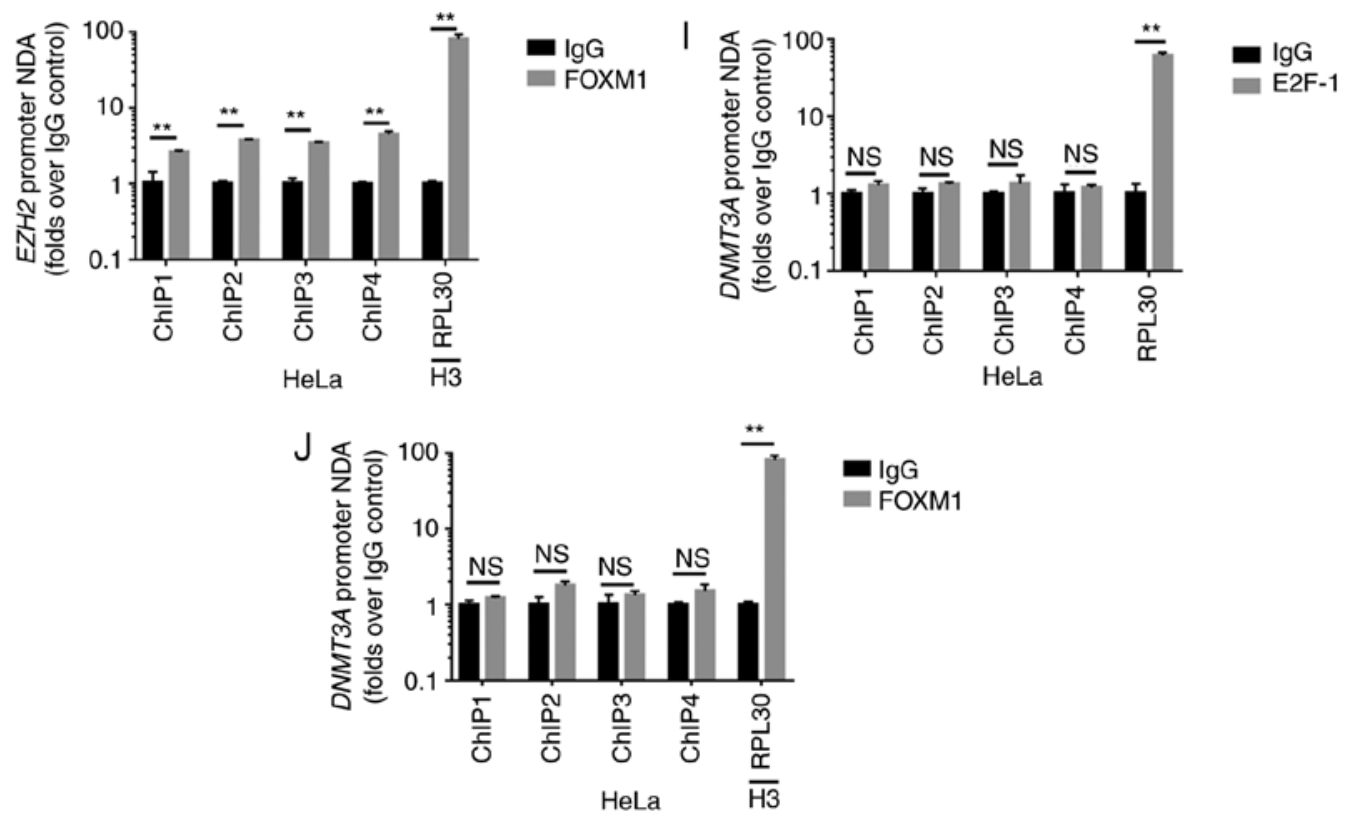

Figure 6. HPV18 oncoprotein E6/E7 alters the expression EZH2 and H3K27me3 in cervical cancer cells. (A and B) C33A cells were transfected with 18E6/E7/E6/7 lentiviral vector and HeLa cells were transfected with 18E6/E7/E6/7 specific siRNAs and western blot analysis was then used to detect EZH2 and H3K27me3 expression. (C) EZH2 and H3K27me3 expression in HeLa and C33A cells detected by western blot analysis. (D) Western blot analysis of the HPV18 E6/E7 protein level in HeLa cells following transfection with siRNAs against EZH2. (E) Co-IP assay was used to analyzed the incorporation between EZH2 and HPV18 E6/E7. (F) Schematic representation of the 4 regions of the EZH2 promoter amplified in the chromatin immunoprecipitation (ChIP)-quantitative PCR (qPCR) experiment. (G and H) Chromatin was cross-linked, fragmented and immunoprecipitated with either IgG (mock) or anti-E2F-1 and anti-FOXM1 ChIP-grade antibody and the purified DNA was used to amplify with respective primer pairs for indicated four regions in the $E Z H 2$ promoters in qPCR. The enrichment of E2F-1 and FOXM1 on EZH2 promoter relative to IgG in HeLa cells, H3 against RPL30 used as positive control. (I and J) Chromatin was cross-linked, fragmented and immunoprecipitated with either IgG (mock) or anti-E2F-1 and anti-FOXM1 ChIP-grade antibody and the purified DNA was used to amplify with respective primer pairs for the indicated 4 regions in the DNMT3A promoters in qPCR. The enrichment of E2F-1 and FOXM1 on DNMT3A promoter relative to IgG in HeLa cells, $\mathrm{H} 3$ against $R P L 30$ used as a positive control. ${ }^{* *} \mathrm{P}<0.01$; ns, not significant.

Tim-3/galectin-9 expression was promoted and the methylation levels of the CpG island in the HAVCR2/LGALS9 promoters were reduced when DNMT3A was knocked down. ChIP analysis revealed that DNMT3A directly bound to the HAVCR2/LGALS9 promoter regions in HeLa cells.
EZH2 is an essential catalytic subunit of PRC2, which silences gene expression by generating a methylated epigenetic mark at H3K27me3 $(27,45,46)$. DNMT3A cooperates with EZH2, and H3K27me3 contributes to the transcriptional regulation of gene expression (47-49). A number of epigenetic 


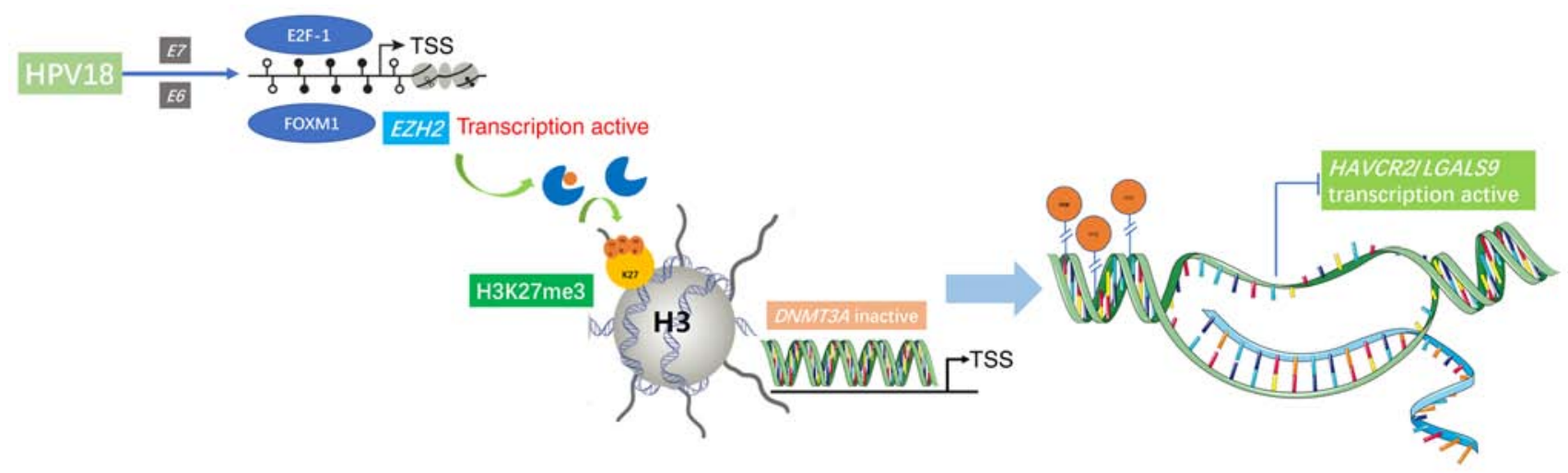

Figure 7. The HPV18 pathway regulates Tim-3/galectin-9 through EZH2-H2K27me3-DNMT3A. HPV18 E6 and E7 regulates EZH2 through direct binding of FOXM1 and E2F-1 the EZH2 promoter in order to activate EZH2 expression; in turn, H3K27me3 is upregulated. EZH2 and H3K27me3 directly interact with the DNMT3A promoter to downregulate its expression. When DNMT3A is decreased, the HAVCR2/LGALS9 promoter region is also demethylated and Tim-3/galectin-9 expression is upregulated.

alterations occur in cellular genomes during HPV-associated carcinogenesis, and these alterations include histone modifications (50-53). Some researchers have also demonstrated that EZH2, H3K27me3 and DNMT1 cooperatively orchestrate epigenetic modification of the $w w c l$ gene promoter in breast cancer (54). In this study, we found that EZH2 and H3K27me3 was overexpressed and DNMT3A was expressed at low levels in cervical cancer tissues. Therefore, we hypothesized that EZH2 and H3K27me3 promote DNMT3A expression in cervical cancer. The downregulation of EZH2 inhibited H3K27me3 expression in HeLa cells and was accompanied by increased DNMT3A protein levels, demonstrating that DNMT3A is a downstream target gene of EZH2 and H3K27me3. Both EZH2 and H3K27me3 were enriched at $\mathrm{CpG}$ loci within the DNMT3A gene promoter in HeLa cells, as demonstrated by ChIP assays.

The knockdown of EZH2 and H3K27me3 expression was also associated with an increased $H A V C R 2 / L G A L S 9$ methylation, which in turn caused the downregulation of Tim-3/galectin-9 expression. The EZH2-catalyzed trimethylation of H3K27 may be a prerequisite for promoter DNA methylation by recruiting DNMT3A. EZH2 and H3K27me3 synergistically regulated DNMT3A promoter expression and cooperatively orchestrated the epigenetic modification of the DNMT3A gene promoter.

HPV16 E6 and E7 can promote the secretion of soluble Tim-3 in oropharyngeal squamous cell carcinoma, resulting in a poor patient prognosis (55). Notably, in this study, Tim-3/galectin-9 was expressed at higher levels in HPV-positive cancer tissues than in HPV-negative tissues. Altering the expression of HPV18 oncoproteins E6 and/or E7 also altered the Tim-3/galectin-9 protein expression level and the methylation status of their promoters. It has been suggested that HPV18 E6 and E7 regulate the expression of Tim-3/galectin-9 in cervical cancer by affecting the methylation level of the genes encoding these proteins. HPV18 E6 and E7 induced the downregulation of DNMT3A protein. The expression of Tim-3/galectin-9 was increased, and the methylation level of the $\mathrm{CpG}$ islands in the HAVCR2/LGALS9 promoters was reduced when DNMT3A was knocked down. These results indicate that the HPV18 oncoproteins regulate Tim-3/galectin-9 expression through DNMT3A. However, the co-IP assay found that neither HPV18 E6 nor HPV18 E7 directly interacted with DNMT3A.

The results of this study illustrate that HPV18 E6 and/or E7 can elevate EZH2 expression in cervical cancer cells. In addition, the expression of EZH2 and $\mathrm{H} 3 \mathrm{~K} 27 \mathrm{me} 3$ proteins in cervical cancer tissues was higher than that in normal tissues. However, the co-IP assay found that neither HPV18 E6 nor HPV18 E7 directly interacted with EZH2, and EZH2 knock down did not affect the expression of HPV18 E6 and E7. E6 oncoprotein can induce the expression of FOXM1 through the MZF1/NKX2-1 axis (29). HPV mediates EZH2 expression through the transcriptional activation of the $E Z H 2$ promoter via E7-mediated release of E2F factors from inhibitory pocket proteins (56). The most well-studied cellular target of the viral oncogene $\mathrm{E} 7$ is $\mathrm{pRb}$, and $\mathrm{E} 2 \mathrm{~F}-1$ is the main target that is regulated by pRb. HPV E7 oncoprotein also leads to increased gene expression through the direct binding of the E2F-1 transcription factor to the gene promoter (30,57-59). EZH2 alterations in HPV16 E6/E7 HFKs lead to a loss of H3K27me3 and to the transcriptional depression of H3K27me3-targeted HOX genes (51). In this study, we used a ChIP assay to illustrate that the transcription factors FOXM1 and E2F-1 can directly bind to the promoter of $E Z H 2$, but do not directly bind to the promoter of DNMT3A. Therefore, we assert the preliminary conclusion that EZH2 and $\mathrm{H} 3 \mathrm{~K} 27 \mathrm{me} 3$ expression is regulated by HPV18 E6 and E7 through the transcription factors FOXM1 and E2F-1.

Our data reveal a key role for EZH2-H3K27me3-DNMT3A in regulating the costimulatory molecules Tim-3/galectin-9 in cervical cancer that involves the HPV18 oncoproteins E6/E7 (Fig. 7). EZH2 and H3K27me3 may represent therapeutic targets, and an epigenetic agent or inhibitor aimed at these proteins could decrease Tim-3/galectin-9 expression. The inhibition of the expression of EZH2 and H3K27me3 may be an effective approach with which to augment the efficacy of negative costimulatory molecules against cervical cancer. DNMT1 and DNMT3B are also associated with the methylation of genes. In this study, we focused on the role of DNMT3A in the regulation of Tim-3 and galectin-9, which is another limitation of this study; therefore, in the future, we aim to study the role of DNMT1 and DNMT3B in the regulation of Tim-3 and galectin-9 expression. 


\section{Acknowledgements}

The authors would like to thank all the teachers at the Center for Translational Medicine of the First Affiliated Hospital of Xi'an Jiaotong University for providing technical assistance.

\section{Funding}

This research was supported by the National Natural Science Foundation of China (no. 81472428), a Fundamental Research Funds for the Central Universities and Nutrition Asia Research Grant by BASF and National Natural Science Foundation of China (grant nos. 81672350, 81872225).

\section{Availability of data and materials}

The dataset analyzed during the current study are publicly available from the online database: GEPIA database (http:/gepia.cancer-pku.cn/).

\section{Authors' contributions}

LZ, ST and YJ conducted the experiments. LZ, LS, MZ, LW, JZ and XY participated in the data analysis. LZ, LS, TY and XY designed the experiments. MZ, LW, MP, YJ, TY and JZ collected the samples from the cervical cancer patients. LZ, ST, TY, JZ and XY wrote and edited the manuscript. All authors have read and approved the final manuscript.

\section{Ethics approval and consent to participate}

The study was approved by the Ethics Committee of the First Affiliated Hospital of Xi'an Jiaotong University (G-272) in Shaanxi, China. Written informed consent was obtained from all patients to participate in this study.

\section{Patient consent for publication}

Not applicable.

\section{Competing interests}

The authors declare that they have no competing interests.

\section{References}

1. Senkomago V, Duran D, Loharikar A, Hyde TB, Markowitz LE, Unger ER and Saraiya M: CDC activities for improving implementation of human papillomavirus vaccination, cervical cancer screening, and surveillance worldwide. Emerg Infect Dis 23, 2017.

2. Fader AN: Surgery in cervical cancer. N Engl J Med 379: 1955-1957, 2018

3. Jiang X, Tang $\mathrm{H}$ and Chen T: Epidemiology of gynecologic cancers in China. J Gynecol Oncol 29: e7, 2018.

4. Tsu V and Jeronimo J: Saving the World's Women from Cervical Cancer. The New England J Med 374: 2509-2511, 2016.

5. Chen W, Zheng R, Baade PD, Zhang S, Zeng H, Bray F, Jemal A, Yu XQ and He J: Cancer statistics in China, 2015. CA Cancer J Clin 66: 115-132, 2016.

6. Schlecht NF, Kulaga S, Robitaille J, Ferreira S, Santos M, Miyamura RA, Duarte-Franco E, Rohan TE, Ferenczy A, Villa LL and Franco EL: Persistent human papillomavirus infection as a predictor of cervical intraepithelial neoplasia. JAMA 286: 3106-3114, 2001.
7. Saraiya M, Unger ER, Thompson TD, Lynch CF, Hernandez BY, Lyu CW, Steinau M, Watson M, Wilkinson EJ, Hopenhayn C, et al: US assessment of HPV types in cancers: Implications for current and 9-valent HPV vaccines. J Natl Cancer Inst 107: djv086, 2015.

8. Kahn JA: HPV vaccination for the prevention of cervical intraepithelial neoplasia. N Engl J Med 361: 271-278, 2009.

9. Baumeister SH, Freeman GJ, Dranoff G and Sharpe AH: Coinhibitory pathways in immunotherapy for cancer. Annu Rev Immunol 34: 539-573, 2016.

10. Topalian SL, Taube JM, Anders RA and Pardoll DM: Mechanism-driven biomarkers to guide immune checkpoint blockade in cancer therapy. Nat Rev Cancer 16: 275-287, 2016.

11. Liu F, Liu Y and Chen Z: Tim-3 expression and its role in hepatocellular carcinoma. J Hematol Oncol 11: 126, 2018.

12. de Mingo Pulido A, Gardner A, Hiebler S, Soliman H, Rugo HS, Krummel MF, Coussens LM and Ruffell B: TIM-3 regulates CD103(+) dendritic cell function and response to chemotherapy in breast cancer. Cancer Cell 33: 60-74.e6, 2018.

13. He Y, Cao J, Zhao C, Li X, Zhou C and Hirsch FR: TIM-3, a promising target for cancer immunotherapy. Onco Targets Ther 11: 7005-7009, 2018.

14. Li X, Hu W, Zheng X, Zhang C, Du P, Zheng Z, Yang Y, Wu J, Ji M, Jiang $J$ and Wu C: Emerging immune checkpoints for cancer therapy. Acta Oncol 54: 1706-1713, 2015.

15. Moriyama K, Kukita A, Li YJ, Uehara N, Zhang JQ, Takahashi I and Kukita T: Regulation of osteoclastogenesis through Tim-3: Possible involvement of the Tim-3/galectin-9 system in the modulation of inflammatory bone destruction. Lab Invest 94: 1200-1211, 2014

16. Chou FC, Kuo CC, Chen HY, Chen HH and Sytwu HK: DNA demethylation of the TIM-3 promoter is critical for its stable expression on T cells. Genes Immun 17: 179-186, 2016.

17. Sasidharan Nair V, El Salhat H, Taha RZ, John A, Ali BR and Elkord E: DNA methylation and repressive H3K9 and H3K27 trimethylation in the promoter regions of PD-1, CTLA-4, TIM-3, LAG-3, TIGIT, and PD-L1 genes in human primary breast cancer. Clin Epigenetics 10: 78, 2018.

18. Cao Y, Zhou X, Huang X, Li Q, Gao L, Jiang L, Huang M and Zhou J: Tim-3 expression in cervical cancer promotes tumor metastasis. PLoS One 8: e53834, 2013.

19. Liang M, Ueno M, Oomizu S, Arikawa T, Shinonaga R, Zhang S, Yamauchi A and Hirashima M: Galectin-9 expression links to malignant potential of cervical squamous cell carcinoma. J Cancer Res Clin Oncol 134: 899-907, 2008.

20. Azizmohammadi S, Azizmohammadi S, Safari A, Kaghazian M, Sadrkhanlo M, Behnod V and Seifoleslami M: High-level expression of RIPK4 and EZH2 contributes to lymph node metastasis and predicts favorable prognosis in patients with cervical cancer. Oncol Res 25: 495-501, 2017.

21. Sun J, Ji J, Huo G, Song Q and Zhang X: miR-182 induces cervical cancer cell apoptosis through inhibiting the expression of DNMT3a. Int J Clin Exp Pathol 8: 4755-4763, 2015.

22. Cao D, Zhang S, Zhang Q, Wei X, Zhao M, Ma Q, Li Y, Wang L, Pei M, Yang T, et al: Prevalence of high-risk human papillomavirus infection among women in Shaanxi province of China: A hospital-based investigation. J Med Virol 89: 1281-1286, 2017.

23. Wei X, Zhang S, Cao D, Zhao M, Zhang Q, Zhao J, Yang T, Pei M, Wang L, Li Y and Yang X: Aberrant hypermethylation of SALL3 with HPV involvement contributes to the carcinogenesis of cervical cancer. PLoS One 10: e0145700, 2015.

24. Zhao M, Li Y, Wei X, Zhang Q, Jia H, Quan S, Cao D, Wang L, Yang T, Zhao J, et al: Negative immune factors might predominate local tumor immune status and promote carcinogenesis in cervical carcinoma. Virol J 14: 5, 2017.

25. Livak KJ and Schmittgen TD: Analysis of relative gene expression data using real-time quantitative PCR and the 2(-Delta Delta C(T)) method. Methods 25: 402-408, 2001.

26. Simon JA and Lange CA: Roles of the EZH2 histone methyltransferase in cancer epigenetics. Mutat Res 647: 21-29, 2008.

27. Fu Y, Chen J, Pang B, Li C, Zhao J and Shen K: EZH2-induced $\mathrm{H} 3 \mathrm{~K} 27 \mathrm{me} 3$ is associated with epigenetic repression of the ARHI tumor-suppressor gene in ovarian cancer. Cell Biochem Biophys 71: 105-112, 2015.

28. Ler LD, Ghosh S, Chai X, Thike AA, Heng HL, Siew EY, Dey S, Koh LK, Lim JQ, Lim WK, et al: Loss of tumor suppressor KDM6A amplifies PRC2-regulated transcriptional repression in bladder cancer and can be targeted through inhibition of EZH2. Sci Transl Med 9: eaai8312, 2017. 
29. Chen PM, Cheng YW, Wang YC, Wu TC, Chen CY and Lee $\mathrm{H}$ : Up-regulation of FOXM1 by E6 oncoprotein through the MZF1/NKX2-1 axis is required for human papillomavirusassociated tumorigenesis. Neoplasia 16: 961-971, 2014.

30. Urrego D, Movsisyan N, Ufartes R and Pardo LA: Periodic expression of Kv10.1 driven by $\mathrm{pRb} / \mathrm{E} 2 \mathrm{~F} 1$ contributes to $\mathrm{G} 2 / \mathrm{M}$ progression of cancer and non-transformed cells. Cell Cycle 15: 799-811, 2016.

31. Finzer P, Krueger A, Stohr M, Brenner D, Soto U, Kuntzen C, Krammer PH and Rösl F: HDAC inhibitors trigger apoptosis in HPV-positive cells by inducing the E2F-p73 pathway. Oncogene 23: 4807-4817, 2004.

32. McCance DJ: Human papillomaviruses and cell signaling. Science's STKE 2005: pe29, 2005.

33. Ghosh K, Chatterjee B, Maheswari U, Athifa M and Kanade SR: 4-Nonylphenol-enhanced EZH2 and RNF2 expression, H3K27me3 and H2AK119ub1 marks resulting in silencing of p21(CDKN1A) in vitro. Epigenomics 11: 899-916, 2019.

34. Wang Y, Wu M, Lei Z, Huang M, Li Z, Wang L, Wang L, Cao Q, Han D, Chang Y, et al: Dysregulation of miR-6868-5p/FOXM1 circuit contributes to colorectal cancer angiogenesis. J Exp Clin Cancer Res 37: 292, 2018.

35. Mersakova S, Nachajova M, Szepe P, Kasajova PS and Halasova E: DNA methylation and detection of cervical cancer and precancerous lesions using molecular methods. Tumor Biol 37: 23-27, 2016.

36. Steenbergen RD, Snijders PJ, Heideman DA and Meijer CJ: Clinical implications of (epi)genetic changes in HPV-induced cervical precancerous lesions. Nat Rev Cancer 14: 395-405, 2014.

37. Cicchini L, Blumhagen RZ, Westrich JA, Myers ME, Warren CJ, Siska C, Raben D, Kechris KJ and Pyeon D: High-risk human papillomavirus E7 alters Host DNA methylome and represses HLA-E expression in human keratinocytes. Sci Rep 7: 3633, 2017.

38. Yasmin R, Siraj S, Hassan A, Khan AR, Abbasi R and Ahmad N: Epigenetic regulation of inflammatory cytokines and associated genes in human malignancies. Mediators Inflamm 2015: 201703, 2015.

39. Micevic G, Thakral D, McGeary M and Bosenberg MW: PD-L1 methylation regulates PD-L1 expression and is associated with melanoma survival. Pigment Cell Melanoma Res 32: 435-440, 2018.

40. Zhang T, Wu J, Ungvijanpunya N, Jackson-Weaver O, Gou Y, Feng J, Ho TV, Shen Y, Liu J, Richard S, et al: Smad6 Methylation Represses NFkB activation and periodontal inflammation. J Dent Res 97: 810-819, 2018.

41. Wang Y, Zhao E, Zhang Z, Zhao G and Cao H: Association between Tim 3 and Ga19 expression and gastric cancer prognosis. Oncol Rep 40: 2115-2126, 2018.

42. Hou N, Ma J, Li W, Zhao L, Gao Q and Mai L: T-cell immunoglobulin and mucin domain-containing protein-3 and galectin-9 protein expression: Potential prognostic significance in esophageal squamous cell carcinoma for Chinese patients. Oncol Lett 14: 8007-8013, 2017

43. Komohara Y, Morita T, Annan DA, Horlad H, Ohnishi K, Yamada S, Nakayama T, Kitada S, Suzu S, Kinoshita I, et al: The Coordinated Actions of TIM-3 on cancer and myeloid cells in the regulation of tumorigenicity and clinical prognosis in clear cell renal cell carcinomas. Cancer Immunol Res 3: 999-1007, 2015.

44. Liang T, Wang X, Wang F, Feng E and You G: Galectin-9: A predictive biomarker negatively regulating immune response in glioma patients. World Neurosurg: Aug 27, 2019 (Epub ahead of print). doi: 10.1016/j.wneu.2019.08.117.

45. Zhou T, Sun Y, Li M, Ding Y, Yin R, Li Z, Xie Q, Bao S and Cai W: Enhancer of zeste homolog 2-catalysed H3K27 trimethylation plays a key role in acute-on-chronic liver failure via TNF-mediated pathway. Cell Death Dis 9: 590, 2018.
46. Sharma V, Malgulwar PB, Purkait S, Patil V, Pathak P, Agrawal R, Kulshreshtha R, Mallick S, Julka PK, Suri A, et al: Genome-wide ChIP-seq analysis of EZH2-mediated H3K27me3 target gene profile highlights differences between low- and high-grade astrocytic tumors. Carcinogenesis 38: 152-161, 2017.

47. Cui H, Hu Y, Guo D, Zhang A, Gu Y, Zhang S, Zhao C, Gong P, Shen X, Li Y, et al: DNA methyltransferase 3A isoform b contributes to repressing E-cadherin through cooperation of DNA methylation and $\mathrm{H} 3 \mathrm{~K} 27 / \mathrm{H} 3 \mathrm{~K} 9$ methylation in EMT-related metastasis of gastric cancer. Oncogene 37: 4358-4371, 2018.

48. Mochizuki D, Misawa Y, Kawasaki H, Imai A, Endo S, Mima M, Yamada S, Nakagawa T, Kanazawa T and Misawa K: Aberrant epigenetic regulation in head and neck cancer due to distinct EZH2 overexpression and DNA hypermethylation. Int J Mol Sci 19: E3707, 2018.

49. Manzo M, Wirz J, Ambrosi C, Villasenor R, Roschitzki B and Baubec T: Isoform-specific localization of DNMT3A regulates DNA methylation fidelity at bivalent $\mathrm{CpG}$ islands. EMBO J 36: 3421-3434, 2017.

50. Hyland PL, Mcdade SS, Mccloskey R, Dickson GJ, Arthur K, McCance DJ and Patel D: Evidence for alteration of EZH2, BMI1, and KDM6A and epigenetic reprogramming in human papillomavirus type 16 E6/E7-expressing keratinocytes. J Virol 85: 10999-11006, 2011.

51. Lindsay CD, Kostiuk MA, Harris J, O'Connell DA, Seikaly H and Biron VL: Efficacy of EZH2 inhibitory drugs in human papillomavirus-positive and human papillomavirus-negative oropharyngeal squamous cell carcinomas. Clin Epigenetics 9: 95, 2017.

52. Biron VL, Mohamed A, Hendzel MJ, Alan Underhill D and Seikaly H: Epigenetic differences between human papillomavirus-positive and -negative oropharyngeal squamous cell carcinomas. J Otolaryngol Head Neck Surg 41 (Suppl 1): S65-S70, 2012.

53. Leonard S, Pereira M, Fox R, Gordon N, Yap J, Kehoe S, Luesley D, Woodman C and Ganesan R: Over-expression of DNMT3A predicts the risk of recurrent vulvar squamous cell carcinomas. Gynecol Oncol 143: 414-420, 2016.

54. Liu X, Li C, Zhang R, Xiao W, Niu X, Ye X, Li Z, Guo Y, Tan J and Li Y: The EZH2- H3K27me3-DNMT1 complex orchestrates epigenetic silencing of the wwcl gene, a Hippo/YAP pathway upstream effector, in breast cancer epithelial cells. Cell Signal 51: 243-256, 2018

55. Hladikova K, Partlova S, Koucky V, Boucek J, Fonteneau JF, Zabrodsky M, Tachezy R, Grega M, Špíšek R and Fialová A: Dysfunction of HPV16-specific CD8 ${ }^{+} \mathrm{T}$ cells derived from oropharyngeal tumors is related to the expression of Tim-3 but not PD-1. Oral Oncol 82: 75-82, 2018.

56. Holland D, Hoppe-Seyler K, Schuller B, Lohrey C, Maroldt J, Dürst M and Hoppe-Seyler F: Activation of the enhancer of zeste homologue 2 gene by the human papillomavirus E7 oncoprotein. Cancer Res 68: 9964-9972, 2008.

57. Yeo-Teh NSL, Ito Y and Jha S: High-risk human papillomaviral oncogenes E6 and E7 target key cellular pathways to achieve oncogenesis. Int J Mol Sci 19: E1706, 2018.

58. Vuillier C, Lohard S, Fetiveau A, Allegre J, Kayaci C, King LE, Braun F, Barillé-Nion S, Gautier F, Dubrez L, et al: E2F1 interacts with BCL-xL and regulates its subcellular localization dynamics to trigger cell death. EMBO Rep 19: 234-243. 2018.

59. Youn HS, Kim TY, Park UH, Moon ST, An SJ, Lee YK, Hwang JT, Kim EJ and Um SJ: Asxl1 deficiency in embryonic fibroblasts leads to cellular senescence via impairment of the AKT-E2F pathway and Ezh2 inactivation. Sci Rep 7: 5198, 2017.

This work is licensed under a Creative Commons Attribution-NonCommercial-NoDerivatives 4.0 International (CC BY-NC-ND 4.0) License. 